\title{
Complexities and bifurcations induced by drug responses in a pulsed tumour-immune model
}

\author{
Jin Yang*, Yuanshun Tan \\ College of Mathematics and Statistics, Chongqing Jiaotong University, \\ Chongqing, 400074,P.R.China, \\ *seehom@126.com \\ Robert A. Cheke \\ Natural Resources Institute, University of Greenwich at Medway, Central Avenue, \\ Chatham Maritime, Chatham, Kent, ME4 4TB, UK
}

\begin{abstract}
Drug response plays a key role in exploring how drug toxicity affects the evolution of tumour cells. Depending on the tumour size in tissue, we initiate pulsed comprehensive therapies, and then construct an impulsive tumour-immune model, in which whether comprehensive therapies applied or not only relies on the threshold of tumour size. By employing the definitions and properties of the Poincare map, we show that the effector cell eradication periodic solution is globally stable under threshold conditions. In the light of the bifurcation theorems, it is shown that transcritical bifurcations can occur with respect to many treatment parameters including depletion rate, chemotherapeutic drug concentration, medicine toxicity coefficient and accumu-

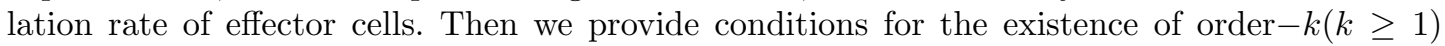
periodic solutions. The results indicate that threshold $R_{0}$ is sensitive to treatment parameters and the proposed system exists with very complex dynamics when treatment parameters are chosen as bifurcation parameters. Moreover, the therapeutic protocol with a smaller chemotherapy drug dosage and more frequent intakes is more effective to maintain higher tumour cell depletion rate.
\end{abstract}

Keywords: Tumour-immune model; Drug response; Poincaré map; Bifurcation.

\section{Introduction}

With the industrialization degree being increasingly raised, people suffer from the pains and damages brought by various types of diseases such as cancer while they enjoy the material civilization brought by speedily developing productivity. How to cure cancer has always been a research hotspot. From early surgical treatment to subsequent radiotherapy and chemotherapy, and to the recently proposed immunotherapy, people have been exploring various therapies for curing cancer [Gubin et al., 2014; Ribas et al., 2003; Dearnaley et al., 1999; Finkelstein \& Fishman, 2012]. In order to evaluate the effectiveness of various treatments and provide theoretical basis for clinical treatment, many tumour-immune dynamic models with different therapies have been proposed and studied.

In 1994, Kuznetsov constructed a two ordinary differential equations to model the interactions between tumour cells and immune cells, the results revealed the mechanisms of tumor dormancy and how the 
immune response being stimulated by tumours [Kuznetsov et al., 1994]. Later, Kirschner and Panetta extended this work by incorporating immunotherapy into the model, they studied the effects of continuous injections of interleukin-2 (IL-2) on the evolution of tumours, and then provided conditions for short-term oscillations and long-term relapse for tumour cells [Kirschner \& Panetta, 1998]. De Pillis proposed and analysed a mathematical model considering immunotherapy and chemotherapy, it was pointed out that the mixed therapies were able to eliminate the entire tumour than a single therapy [de Pillis et al., 2006]. Shu studied a delay tumour-immune model with immune response to show the phenomena of oscillations for tumours [Shu et al., 2014].

All these studies have emphasized the role of immunotherapy and chemotherapy in the control of tumours. In reality, the pulsed therapies are often used to cure cancers, which are confirmed by experiments and clinics [Hegmans et al., 2005; Yamaguchi et al., 2006; Duffey et al., 2004; Walther et al., 1999]. Thus, many tumour-immune models with pulsed therapies have been proposed and analysed. Wei and Yang constructed tumour-immune models in the case of immunotherapy and chemotherapy applied at different fixed moments [Walther \& Lin, 2013; Yang et al., 2015, 2019a,b]. They not only derived conditions for the tumour free periodic solution, but also studied the persistence of the system, it was shown that the higher the frequencies of Adoptive Cellular Immunotherapy (ACI), the better the treatment for cancer. Furthermore, Duffey pointed out that there is a closed-loop control mechanism for cancer therapy, which means that comprehensive therapy should only be carried out once the tumour size reaches a threshold value (denoted by $T_{L}$ ) [Duffey et al., 2004]. Based on the tumour threshold value $T_{L}$, Tang and co-authors proposed a piecewise tumour-immune model with effector cell-guided therapy [Tang et al., 2015, 2017], the results suggested that the tumours could either grow unrestrainedly or be controlled below a threshold level or stabilized at a constant. Moreover, they further derived an impulsive tumour-immune model with closed-loop interventions to study how combinations of surgery with immunotherapy affected the evolution of tumours. [Tang et al., 2016]. They pointed out that a single surgery could only maintain the tumour size below a threshold, but comprehensive therapy not only maintained the tumour size below a threshold, but also retained the activity of immune system.

These studies were assumed that the killing rates caused by chemical control (or the instantaneous rate of resection for tumour cells caused by surgery) are constants, which were unreasonable in practice. In fact, when the tumours undergo certain stages of development, on the one hand, chemotherapy can kill a fraction of tumours with a maximum less than one, on the other hand, the killing fraction may mainly depend on the types of the chemotherapy drugs and dosages [Pazdur et al., 2005]. Therefore, based on the tumour threshold value $T_{L}$ during treatment and then by employing theory of the impulsive semi-dynamical systems [Simeonov \& Bainov , 1988; Bainov \& Simeonov , 1993], we propose an impulsive tumour-immune model with drug response of chemotherapy as nonlinear pulses to model the closed-loop treatments, and we will focus on the following questions: (1) How do control parameters including the depletion rate of drug toxicity, the concentration of chemotherapy drug, medicine toxicity coefficient and the accumulation rate affect the evolution of tumours? (2) How to implement chemotherapy and immunotherapy to better treat tumours? (3) How do comprehensive therapy affects the dynamics of the proposed impulsive tumourimmune model?

The paper is arranged as follows. We will derive the mathematical model in section 2.1 and then provide some useful definitions and important lemmas of impulsive semi-dynamical systems in section 2.2. In section 3, we not only construct the Poincaré map, but also discuss its main properties. In section 4, we first deduce the expression of effector cell eradication periodic solution. Then the transcritical bifurcations with respect to key control parameters are studied. Moreover, we give threshold conditions for the existence of order- $k(k \geq 1)$ periodic solutions. In section 5 , sensitivity analyses and bifurcation analyses are performed to answer the proposed questions, and we also address the biological implications for cancer treatment. At last, conclusions are obtained. 


\section{Mathematical model}

\subsection{Model formation}

To describe the interactions between tumour cells and immune cells, Kuznetsov proposed a two ordinary differential equations which are usually of predator-prey type [Kuznetsov et al., 1994]. Let $x(t)$ and $y(t)$ be the densities of tumour cells and effector cells at time $t$, respectively. Then the model derived by Kuznetsov can be formulated by following system

$$
\left\{\begin{array}{l}
\frac{d x(t)}{d t}=r x(t)\left(1-\frac{x(t)}{K}\right)-a x(t) y(t), \\
\frac{d y(t)}{d t}=\frac{b x(t) y(t)}{1+w x(t)}-c x(t) y(t)-d y(t),
\end{array}\right.
$$

where $r$ is the intrinsic growth rate of tumour cells, $K$ represents carrying capacity of tumour cells, $a$ is the killing rate of tumour cells due to effector cells, $c$ is the killing rate of effector cells due to tumour cells, $d$ is the death rate of effector cells, $b$ denotes the maximum immune response rate due to presence of tumour cells and $w$ is the steepness of immune response.

Inspired by idea of closed-loop treatment, in the light of the tumour threshold value $T_{L}$ we propose a nonlinear pulsed model (1) concerning both chemotherapy and immunotherapy, which are formulated by following system

$$
\left\{\begin{aligned}
\frac{d x(t)}{d t} & =r x(t)\left(1-\frac{x(t)}{K}\right)-a x(t) y(t), \\
\frac{d y(t)}{d t} & =\frac{b x(t) y(t)}{1+w x(t)}-c x(t) y(t)-d y(t), \\
x\left(t^{+}\right) & =\left(1-K_{1}\left(C_{0}\right)\right) x(t), \\
y\left(t^{+}\right) & =\left(\left(1-K_{2}\left(C_{0}\right)\right) y(t)\right) \theta+\tau,
\end{aligned}\right\} \quad x(t)=T_{L},
$$

where $T_{L}$ is the threshold number of tumour cells at which chemotherapy is needed to be applied. In reality, the chemotherapy drug kills both of tumour cells and effector cells, but that the kill rates differ for tumour cells and effector cells, and we assume that the response curves satisfy the exponential functions [Panetta , 1996]. $K_{1}\left(C_{0}\right)$ and $K_{2}\left(C_{0}\right)$ are killing rates of tumour cells and effector cells due to chemotherapy which can be described by

$$
K_{1}\left(C_{0}\right)=d_{T}\left(1-\exp \left(-k_{1} C_{0}\right)\right), K_{2}\left(C_{0}\right)=d_{E}\left(1-\exp \left(-k_{2} C_{0}\right)\right),
$$

where $d_{T}\left(d_{E}\right)$ is the rate of tumour (effector) cell depletion of drug toxicity, $C_{0}$ is the concentration of chemotherapy drug in the blood and $k_{1}\left(k_{2}\right)$ is medicine toxicity coefficient to tumour cells (effector cells). It is assumed that $K_{1}, K_{2} \in[0,1)$ to keep all solutions of system (2) from being negative. $\theta>0$ denotes the accumulation rate of effector cells due to an external input of IL-2. $\tau>0$ is the amount of injected ACI such as lymphokine-activated killer (LAK) and tumour infiltrating lymphocyte (TIL) cells. We let $x\left(0^{+}\right)$and $y\left(0^{+}\right)$be the initial densities of tumour cells and effector cells, and then assume the initial density of tumour cells to be less than the threshold $T_{L}$. If the tumour size reaches threshold $T_{L}$, then the comprehensive therapies including chemotherapy and immunotherapy are implemented, and thereby the amount of tumour cells and effector cells is updated to $\left(1-K_{1}\left(C_{0}\right)\right) T_{L}$ and $\left(\left(1-K_{2}\left(C_{0}\right)\right) y(t)\right) \theta+\tau$.

\subsection{Preliminaries}

Consider the following generalized planar impulsive semi-dynamical systems [Simeonov \& Bainov , 1988; Bainov \& Simeonov, 1993]:

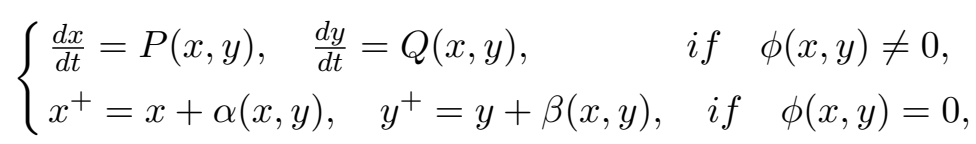


where $x^{+}=x\left(t^{+}\right), y^{+}=y\left(t^{+}\right)$and $(x, y) \in R^{2} . P, Q, \alpha, \beta$ are continuous functions from $R^{2}$ to $R$. Let $\mathcal{I} \subset R^{2}$ be the impulsive set. For any $Z(x, y) \in \mathcal{I}$, the impulsive map $I: R^{2} \longrightarrow R^{2}$ is defined by

$$
I(z)=(x+\alpha(x, y), y+\beta(x, y))=\left(x^{+}, y^{+}\right) \in R^{2},
$$

and denote $Z^{+}=\left(x^{+}, y^{+}\right)$as impulsive point of $Z$.

Define $\mathcal{P}=I(\mathcal{I})$ and $\mathcal{P}$ is said to be the phase set. $\left(R^{2}, \Pi\right)$ is defined as a semi-dynamical system [Ciesielski , 2004; Kaul , 1990]. For any $Z \in R^{2}$, the positive solution initiating from $Z$ is described by $C^{+}(Z)=\left\{\Pi(Z, t) \mid t \in R^{+}\right\}$, and we denote $C^{+}(Z)$ as $\Pi^{+}(Z)$. Further, Let $G(Z, t)=\left\{Z^{\prime} \mid \Pi\left(Z^{\prime}, t\right)=Z\right\}$ for any $t \geq 0$ and $Z \in R^{2}$. Concerning these notations, the following useful definitions can be introduced [Ciesielski , 2004; Kaul, 1990].

Definition 1. An impulsive semi-dynamical system $\left(R^{2}, \Pi ; \mathcal{P}, \mathcal{I}\right)$ consists of a continuous semi-dynamical system $\left(R^{2}, \Pi\right)$ together with a nonempty closed subset $\mathcal{I}$ of $X$ and a continuous function $I: \mathcal{I} \longrightarrow R^{2}$ such that for any $Z \in \mathcal{I}$ we have $G(Z, t) \cap \mathcal{P} \neq \varnothing$ and $\Pi(Z, t) \cap \mathcal{I} \neq \varnothing$.

Definition 2. A solution $\Pi^{+}(Z)$ in $\left(R^{2}, \Pi ; \mathcal{P}, \mathcal{I}\right)$ is called order $k$ periodic solution if for two positive integers $m$ and $k$ we have $\mathcal{I}^{m}(Z)=\mathcal{I}^{m+k}(Z)$, where $Z \in \mathcal{P}$ and $k$ is the smallest integer.

Lemma 1. ([Simeonov \& Bainov, 1988; Bainov \& Simeonov , 1993]) For system (1), the order k-periodic solution $(x, y)=(\xi(t), \eta(t))$ is orbitally asymptotically stable and enjoys the property of asymptotic phase if the Floquet multiplier $\mu_{2}$ satisfies $\left|\mu_{2}\right|<1$, where

$$
\mu_{2}=\prod_{k=1}^{q} \Delta_{k} \exp \left(\int_{0}^{T}\left[\frac{\partial P}{\partial x}(\xi(t), \eta(t))+\frac{\partial Q}{\partial y}(\xi(t), \eta(t))\right] d t\right)
$$

with

$$
\Delta_{k}=\frac{P_{+}\left(\frac{\partial \beta}{\partial y} \frac{\partial \phi}{\partial x}-\frac{\partial \beta}{\partial x} \frac{\partial \phi}{\partial y}+\frac{\partial \phi}{\partial x}\right)+Q_{+}\left(\frac{\partial \alpha}{\partial x} \frac{\partial \phi}{\partial y}-\frac{\partial \alpha}{\partial y} \frac{\partial \phi}{\partial x}+\frac{\partial \phi}{\partial y}\right)}{P \frac{\partial \phi}{\partial x}+Q \frac{\partial \phi}{\partial y}},
$$

and function $\phi$ is continuously differentiable with respect to $x, y .(x, y) \notin \mathcal{I}$ is equivalent to $\phi(x, y) \neq 0$. $P, Q, \partial \alpha / \partial x, \partial \alpha / \partial y, \partial \beta / \partial x, \partial \beta / \partial y, \partial \phi / \partial x$ and $\partial \phi / \partial y$ can be calculated at the point $\left(\xi\left(t_{k}\right), \eta\left(t_{k}\right)\right)$. Denote $P_{+}=P\left(\xi\left(t_{k}^{+}\right), \eta\left(t_{k}^{+}\right)\right)$and $Q_{+}=Q\left(\xi\left(t_{k}^{+}\right), \eta\left(t_{k}^{+}\right)\right) . t_{k}(k, q \in N, N$ is non-negative integers) is the time of the $k$-th jump.

Lemma 2. ([Rasband, 1990]) Let $F: R \rightarrow R$ be a one-parameter family of $C^{2}$ map satisfying

(1) $F(0, \rho)=0$ for all $\rho$; (2) $\frac{\partial F}{\partial x}(0,0)=1$;

(3) $\frac{\partial^{2} F}{\partial x \partial \rho}(0,0)>0$; (4) $\frac{\partial^{2} F}{\partial x^{2}}(0,0)<0$.

Then for $\rho_{1}<0<\rho_{2}$ and any $\varepsilon>0$ we have (i) if $\rho_{1}<\rho<0$, then $F_{\rho}$ has two fixed points 0 and $\rho^{*}>0 \in(-\varepsilon, \varepsilon)$ with 0 being asymptotically stale and $\rho^{*}$ being unstable. (ii) if $0<\rho<\rho_{2}$, then $F_{\rho}$ has two fixed points 0 and $\rho^{*}<0 \in(-\varepsilon, \varepsilon)$ with 0 being unstable and $\rho^{*}$ being asymptotically stale.

In order to study the global dynamics of system (2) and further to address their biological implications with respect to cancer treatment, we first need to discuss all possible dynamics of system (1). The vertical isocline of system (1) which plays a key role in defining the domain of impulsive set is denoted as $L_{1}$,

$$
L_{1}: y=\frac{r}{a}\left(1-\frac{x}{K}\right) .
$$

System (1) has a trivial equilibrium $E_{0}(0,0)$, a boundary equilibrium or effector cell eradication equilibrium $E_{K}(K, 0)$, and two interior equilibria $E_{1,2}\left(x_{1,2}, y_{1,2}\right)$ under certain conditions where

$$
x_{1,2}=\frac{b-c-d w \pm \sqrt{(b-c-d w)^{2}-4 c w d}}{2 c w} \text { and } y_{1,2}=\frac{r}{a}\left(1-\frac{x_{1,2}}{K}\right) .
$$

By direct calculation, we have following results [Kuznetsov et al., 1994].

Proposition 1. (i) if $b-c-d w<2 \sqrt{c w d}$ or $b-c-d w \geq 2 \sqrt{c w d}$ and $x_{2} \geq K$, then system (1) exists a trivial equilibrium $E_{0}(0,0)$ and a globally stable boundary equilibrium $E_{K}(K, 0)$;

(ii) if $b-c-d w>2 \sqrt{c w d}$ and $x_{2}<K \leq x_{1}$, then system (1) exists a trivial equilibrium $E_{0}(0,0)$, a boundary equilibrium $E_{K}(K, 0)$ and a globally stable equilibrium $x_{2}$; 
(iii) if $b-c-d w>2 \sqrt{c w d}$ and $x_{1}>K$, then system (1) exists a trivial equilibrium $E_{0}(0,0)$, a local stable boundary equilibrium $E_{K}(K, 0)$, a local stable equilibrium $x_{2}$ and a saddle point $x_{1}$.

In reality, if tumour cells invade into an organism, then tumour cell proliferation will gradually destroy the immune system of the organism. As a result, the tumour size will continuously grow and at last reach its carrying capacity $K$ in the absence of treatments involving chemotherapy and immunotherapy. Therefore, unless otherwise specified we assume that $b-c-d w<2 \sqrt{c w d}$ or $b-c-d w \geq 2 \sqrt{c w d}$ and $x_{2} \geq K$ hold true, in this case system (1) exists a trivial equilibrium $E_{0}$ and a globally stable boundary equilibrium $E_{K}$. Meanwhile, there does not exist interior equilibria $E_{1,2}$ for system (1) and any solution initiating from first quadrant of system (1) will finally tend to the effector cell eradication equilibrium $E_{K}$. The main objective of comprehensive therapy including chemotherapy and immunotherapy is to control tumour cells below a given threshold which should be especially below the carrying capacity, in addition to show how the strength of treatment affects the outcomes of tumour proliferation. Thus, we assume $T_{L}<K$ holds true throughout the paper, and the comprehensive therapy is implemented once the tumour size reaches threshold $T_{L}$.

\section{Poincaré map}

\subsection{Domain of the Poincaré map}

The existence of periodic solutions which implies periodic applications of comprehensive therapy of system (2) is influenced not only by a prescribed threshold level $T_{L}$, but also determined by the strength of comprehensive therapy denoted by killing rates $K_{1}\left(C_{0}\right)$ and $K_{2}\left(C_{0}\right)$, the amount $\tau$ of injected ACI and the accumulation rate $\theta$ of effector cells. Because these key factors related to comprehensive therapy would affect the domains of the Poincaré map and further the existence of its fixed points. So we first need to give the exact domains of the Poincaré map.

For simplicity, we denote by

$$
L_{2}: x=\left(1-K_{1}\left(C_{0}\right)\right) T_{L}, \quad L_{3}: x=T_{L},
$$

where $K_{1}\left(C_{0}\right)$ is defined by equation (3). Due to $0<T_{L}<K$, we denote the intersection point of $L_{1}$ with $L_{3}$ as $Q\left(T_{L}, y_{T_{L}}\right)$, the intersection point of $L_{1}$ with $L_{2}$ is denoted as $P\left(\left(1-K_{1}\left(C_{0}\right)\right) T_{L}, y_{K_{1} T_{L}}\right)$, here

$$
y_{T_{L}}=\frac{r}{a}\left(1-\frac{T_{L}}{K}\right), y_{K_{1} T_{L}}=\frac{r}{a}\left(1-\frac{\left(1-K_{1}\left(C_{0}\right)\right) T_{L}}{K}\right) .
$$

It follows from the definitions of the impulsive semi-dynamical system (1) that

$$
\alpha(x, y)=-K_{1}\left(C_{0}\right) x, \beta(x, y)=\left(\left(1-K_{2}\left(C_{0}\right)\right) \theta-1\right) y+\tau,
$$

the impulsive set is defined by

$$
\mathcal{I}=\left\{(x, y): x=T_{L}, 0 \leq y \leq \frac{r}{a}\left(1-\frac{T_{L}}{K}\right)\right\},
$$

and the impulsive map is denoted as

$$
I\left(T_{L}, y\right)=\left(x^{+}, y^{+}\right)=\left(\left(1-K_{1}\left(C_{0}\right)\right) T_{L},\left(\left(1-K_{2}\left(C_{0}\right)\right) y(t)\right) \theta+\tau\right) .
$$

After one single pulse, we obtain the phase set as follows

$$
\mathcal{P}=I(\mathcal{I})=\left\{\left(x^{+}, y^{+}\right) \mid x^{+}=\left(1-K_{1}\left(C_{0}\right)\right) T_{L}, y^{+} \in \operatorname{Dom}\right\},
$$

where $D o m=\left[\tau,\left(\left(1-K_{2}\left(C_{0}\right)\right) \frac{r}{a}\left(1-\frac{T_{L}}{K}\right)\right) \theta+\tau\right]$. Without loss of generality, we assume that any starting point satisfies $\left(x_{0}^{+}, y_{0}^{+}\right) \in \mathcal{P}$. Based on the domains of the phase set and impulsive set, it enable us to construct the important Poincaré map. 


\subsection{Poincaré map and main properties}

To define the Poincaré map, we choose $L_{2}$ as a Poincaré section. It follows from the globally stability of the equilibrium $E_{K}$ that any solution initiating from point $U_{k}^{+}\left(\left(1-K_{1}\left(C_{0}\right)\right) T_{L}, y_{k}^{+}\right) \in L_{2}$ has a unique intersection point with $L_{4}$ which is denoted by $U_{k+1}\left(T_{L}, y_{k+1}\right)$. Based on the Cauchy-Lipschitz Theorem, we obtain $y_{k+1}=g\left(y_{k}^{+}\right)$which means $y_{k+1}$ only depends on $y_{k}^{+}$. After a single pulse, point $U_{k+1}$ maps to point $U_{k+1}^{+}\left(\left(1-K_{1}\left(C_{0}\right)\right) T_{L},\left(\left(1-K_{2}\left(C_{0}\right)\right) y_{k+1}\right) \theta+\tau\right)$. Thereby, the Poincaré map can be defined as

$$
y_{i+1}^{+}=\left(\left(1-K_{2}\left(C_{0}\right)\right) g\left(y_{i}^{+}\right)\right) \theta+\tau \triangleq \Psi\left(y_{i}^{+}\right) .
$$

The Poincaré map $\Psi\left(y_{i}^{+}\right)$defined by (2) is an abstract expression which implies that it is hard to discuss its properties. Thus, we need to deduce its explicit expression. To do this, we rewrite model (2) as follows

$$
\left\{\begin{array}{l}
\frac{d y}{d x}=\frac{\frac{b x y}{1+w x}-c x y-d y}{r x\left(1-\frac{x}{K}\right)-a x y} \doteq \psi(x, y), \\
y\left(\left(1-K_{1}\left(C_{0}\right)\right) T_{L}\right)=y_{0}^{+} .
\end{array}\right.
$$

Define an important open set as

$$
D=\left\{(x, y) \mid x>0, y>0, y<\frac{r}{a}\left(1-\frac{x}{K}\right)\right\},
$$

then it is obviously that function $\psi(x, y)$ is continuously differentiable in open set $D$. Note that $x_{0}^{+}=$ $\left(1-K_{1}\left(C_{0}\right)\right) T_{L}$, let the vertical component $y_{0}^{+}$of the initial point be $y_{0}^{+} \doteq S<y_{K_{1} T_{L}}$ and $S \in \mathcal{P}$. Define

$$
y(x)=y\left(x ;\left(1-K_{1}\left(C_{0}\right)\right) T_{L}, S\right)=y(x, S), \quad\left(1-K_{1}\left(C_{0}\right)\right) T_{L} \leq x \leq T_{L},
$$

then model (3) becomes

$$
y\left(T_{L}, S\right)=S+\int_{\left(1-K_{1}\left(C_{0}\right)\right) T_{L}}^{T_{L}} \psi(s, y(s, S)) d s .
$$

From (5), the Poincaré map defined by (2) has the following expression

$$
\Psi(S)=\left(\left(1-K_{2}\left(C_{0}\right)\right) y\left(T_{L}, S\right)\right) \theta+\tau .
$$
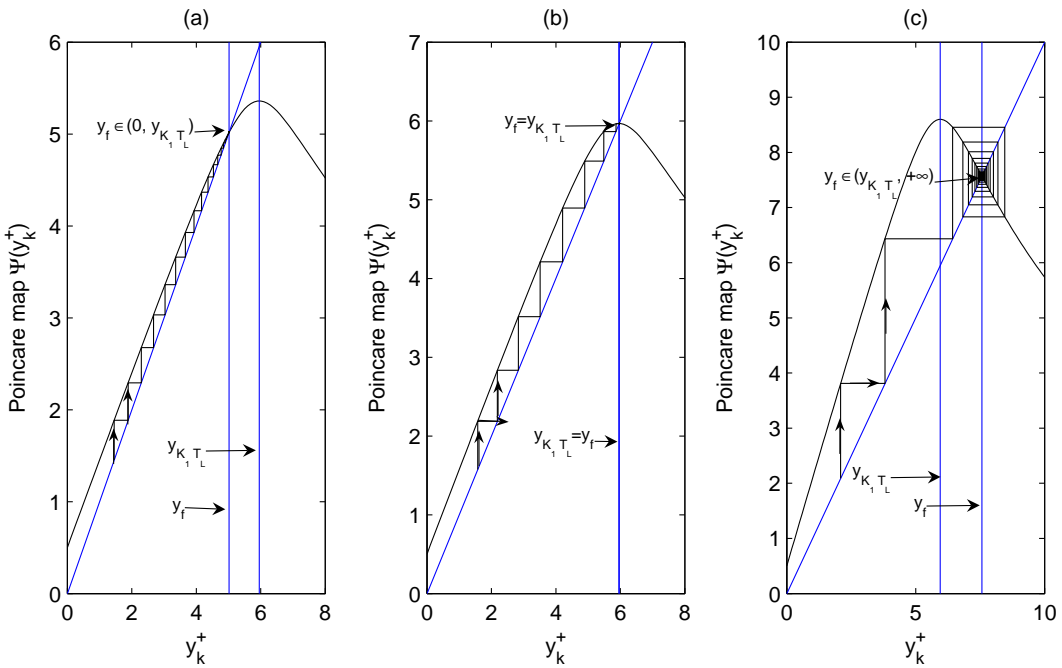

Fig. 1. The fixed point of Poincaré map $\Psi$ with parameters fixed as $r=5, K=100, a=0.8, w=1, b=0.3, c=0.23$, $d=0.3, d_{T}=0.2, d_{E}=0.4, k_{1}=0.5, k_{2}=1, C_{0}=0.65, \tau=0.5$ and $E T=0.5$. (a) $\theta=1.2$; (b) $\theta=1.35$; (c) $\theta=2$. 
Theorem 1. The Poincaré map $\Psi$ has the following main properties:

(I) The range of the Poincaré map $\Psi$ is $[0,+\infty)$ with its domain defined on $\left[\tau, \Psi\left(y_{K_{1} T_{L}}\right)\right]=[\tau,((1-$ $\left.\left.\left.K_{2}\left(C_{0}\right)\right) y\left(\left(1-K_{1}\left(C_{0}\right)\right) T_{L}, y_{K_{1} T_{L}}\right)\right) \theta+\tau\right]$. Meanwhile, $\Psi$ is increasing on $\left[0, y_{K_{1} T_{L}}\right]$ and decreasing on $\left[y_{K_{1} T_{L}},+\infty\right)$.

(II) $\Psi$ is not only continuously differentiable, but also concave on $\left[0, y_{K_{1} T_{L}}\right)$.

(III) $\Psi$ always has a unique fixed point.

(IV) A horizontal asymptote $\Psi=\tau$ exists as $y_{k}^{+} \rightarrow+\infty$.

Proof. (I) Because the boundary equilibrium $E_{K}$ is globally stable, all solutions of system (2) will finally tend to $E_{K}$, which means that any solution initiating from the line $x=\left(1-K_{1}\left(C_{0}\right)\right) T_{L}$ will approach the line $x=T_{L}$. Thereby, $\Psi$ is defined on $[0,+\infty)$. For any $y_{i}^{+}, y_{j}^{+} \in\left[0, y_{K_{1} T_{L}}\right]$ with $y_{i}^{+}<y_{j}^{+}$, the CauchyLipschitz Theorem indicates that the vertical coordinates of the trajectories $\psi\left(t ;\left(1-K_{1}\left(C_{0}\right)\right) T_{L}, y_{i}^{+}\right)$and $\psi\left(t ;\left(1-K_{1}\left(C_{0}\right)\right) T_{L}, y_{j}^{+}\right)$satisfies $y_{i+1}=y\left(t_{1},\left(1-K_{1}\left(C_{0}\right)\right) T_{L}, y_{i}^{+}\right)<y_{j+1}=y\left(t_{2},\left(1-K_{1}\left(C_{0}\right)\right) T_{L}, y_{j}^{+}\right)$with $x\left(t_{1},\left(1-K_{1}\left(C_{0}\right)\right) T_{L}, y_{i}^{+}\right)=x\left(t_{2},\left(1-K_{1}\left(C_{0}\right)\right) T_{L}, y_{j}^{+}\right)=T_{L}$. Due to one time pulse,

$$
\Psi\left(y_{i}^{+}\right)=\left(\left(1-K_{2}\left(C_{0}\right)\right) y_{i+1}\right) \theta+\tau<\left(\left(1-K_{2}\left(C_{0}\right)\right) y_{j+1}\right) \theta+\tau=\Psi\left(y_{j}^{+}\right)
$$

holds such that $\Psi$ is increasing on $\left[0, y_{K_{1} T_{L}}\right]$. Similarly, for any $y_{i}^{+}, y_{j}^{+} \in\left[y_{K_{1} T_{L}},+\infty\right)$ with $y_{i}^{+}<y_{j}^{+}$, since the region above the line $L_{1}$ satisfies $d y / d t<0$ and $d x / d t<0$ and the region below the line $L_{1}$ satisfies $d y / d t<0$ and $d x / d t>0$, the trajectories $\psi\left(t ;\left(1-K_{1}\left(C_{0}\right)\right) T_{L}, y_{i}^{+}\right)$and $\psi\left(t ;\left(1-K_{1}\left(C_{0}\right)\right) T_{L}, y_{j}^{+}\right)$will first meet the isocline $L_{1}$, then across $x=\left(1-K_{1}\left(C_{0}\right)\right) T_{L}$ and finally approach the line $x=T_{L}$ after time $t_{1}$ and $t_{2}$, respectively. Thereby, at times $t_{1}$ and $t_{2}$, the vertical coordinates of these two trajectories satisfy $y_{i+1}=y\left(t_{1},\left(1-K_{1}\left(C_{0}\right)\right) T_{L}, y_{i}^{+}\right)>y_{j+1}=y\left(t_{2},\left(1-K_{1}\left(C_{0}\right)\right) T_{L}, y_{j}^{+}\right)$. After one time pulse, one obtains

$$
\Psi\left(y_{i}^{+}\right)=\left(\left(1-K_{2}\left(C_{0}\right)\right) y_{i+1}\right) \theta+\tau>\left(\left(1-K_{2}\left(C_{0}\right)\right) y_{j+1}\right) \theta+\tau=\Psi\left(y_{j}^{+}\right),
$$

it suggests that $\Psi$ is decreasing on $\left[y_{P},+\infty\right)$ with its range defined on $\left[\tau,\left(\left(1-K_{2}\left(C_{0}\right)\right) y((1-\right.\right.$ $\left.\left.\left.\left.K_{1}\left(C_{0}\right)\right) T_{L}, y_{K_{1} T_{L}}\right)\right) \theta+\tau\right]$.

(II) To show that $\Psi$ is both continuous and differentiable, we focus on two cases: $y<y_{K_{1} T_{L}}$ and $y \geq y_{K_{1} T_{L}}$. Let

$$
M(x, y)=r x\left(1-\frac{x}{K}\right)-a x y, \quad N(x, y)=\frac{b x y}{1+w x}-c x y-d y .
$$

If $y<y_{K_{1} T_{L}}$, then $M(x, y) \neq 0$ for any $(x, y) \in\left\{\left(1-K_{1}\left(C_{0}\right)\right) T_{L} \leq x \leq T_{L}, 0 \leq y<y(t,(1-\right.$ $\left.\left.\left.K_{1}\left(C_{0}\right)\right) T_{L}, y_{K_{1} T_{L}}\right)\right\} \doteq \Upsilon_{(x, y)}$. Note that $\psi(x, y)=N(x, y) / M(x, y)$, then

$$
\frac{\partial \psi}{\partial y}=\frac{r x\left(1-\frac{x}{K}\right)(b x-(c x+d)(1+w x))}{(1+w x)\left(r x\left(1-\frac{x}{K}\right)-a x y\right)^{2}} .
$$

Thus $\partial \psi / \partial y$ is continuous on $\Upsilon_{(x, y)}$ which means that $\Psi$ is continuously differential on $\left[0, y_{K_{1} T_{L}}\right)$. If $y_{0}^{+} \geq y_{K_{1} T_{L}}$, then any trajectories starting from $\left(\left(1-K_{1}\left(C_{0}\right)\right) T_{L}, y_{0}^{+}\right)$will turn around point $P((1-$ $\left.\left.K_{1}\left(C_{0}\right)\right) T_{L}, y_{K_{1} T_{L}}\right)$ and then across line $x=\left(1-K_{1}\left(C_{0}\right)\right) T_{L}$ at point $\left(\left(1-K_{1}\left(C_{0}\right)\right) T_{L}, \bar{y}_{0}^{+}\right)\left(\bar{y}_{0}^{+}<y_{K_{1} T_{L}}\right)$, and meet $x=T_{L}$ in a finite time. We denote the map $\bar{\omega}: \bar{\omega}\left(y_{0}^{+}\right)=\bar{y}_{0}^{+}$, and $\bar{\omega}$ is a continuously differentiable function in the light of the standard theory of Poincare application. Moreover, $\Psi$ is the composite function of $\Psi$ and $\bar{\omega}$ for any $y \in\left[0, y_{K_{1} T_{L}}\right)$. Due to the fact that $\Psi$ is continuously differential on $\left[0, y_{K_{1} T_{L}}\right)$ and $\bar{\omega}$ is also continuously differential, we know that $\Psi$ is continuously differential on $\left[y_{K_{1} T_{L}},+\infty\right)$.

Now we show that $\Psi$ is concave on $\left[0, y_{K_{1} T_{L}}\right)$. First of all, on $\left[0, y_{K_{1} T_{L}}\right)$ equation (7) holds, further,

$$
\frac{\partial^{2} \psi}{\partial y^{2}}=\frac{2 r a x^{2}\left(1-\frac{x}{K}\right)(b x-(c x+d)(1+w x))}{(1+w x)\left(r x\left(1-\frac{x}{K}\right)-a x y\right)^{3}} .
$$

In the light of $y<y_{K_{1} T_{L}}$, we obtain $r x\left(1-\frac{x}{K}\right)-a x y>0$ and $b x-(c x+d)(1+w x)<0$ provided $b-c-d w<2 \sqrt{c w d}$. So $\partial \psi / \partial y<0$ and $\partial^{2} \psi / \partial y^{2}<0$. Therefore,

$$
\frac{\partial y(x, S)}{\partial S}=\exp \left[\int_{\left(1-K_{1}\left(C_{0}\right)\right) T_{L}}^{x} \frac{\partial \psi}{\partial y} d z\right]>0
$$


and

$$
\frac{\partial^{2} y(x, S)}{\partial S^{2}}=\frac{\partial y(x, S)}{\partial S} \int_{\left(1-K_{1}\left(C_{0}\right)\right) T_{L}}^{x} \frac{\partial^{2} \psi}{\partial y^{2}} \frac{\partial y(x, S)}{\partial S} d z<0 .
$$

According to (5) and (6), the Poincaré map $\Psi$ is concave on $\left[0, y_{K_{1} T_{L}}\right.$ ) (Fig.1).

(III) From the above analysis, we have proved that the Poincaré map $\Psi(y)$ is increasing on $\left[0, y_{K_{1} T_{L}}\right.$ ) and decreasing on $\left[y_{K_{1} T_{L}},+\infty\right)$. So for any $y^{I} \in\left[0, y_{K_{1} T_{L}}\right)$ and $y^{I I} \in\left[y_{K_{1} T_{L}},+\infty\right)$ we obtain $\Psi\left(y^{I}\right)>y^{I}$ and $\Psi\left(y^{I I}\right)<y^{I I}$. If $\Psi\left(y_{K_{1} T_{L}}\right)<y_{K_{1} T_{L}}$, note that $\Psi(0)=\tau>0$, then the intermediate value theorem of continuous functions indicates that the Poincaré map $\Psi$ exists at least one fixed point $y_{f}$ and $0<y_{f}<y_{K_{1} T_{L}}$ (Fig.1(a)). If $\Psi\left(y_{K_{1} T_{L}}\right) \geq y_{K_{1} T_{L}}$, on $\left[y_{K_{1} T_{L}},+\infty\right)$ we have $\Psi\left(y^{I I}\right)<y^{I I}$, then the Poincaré map $\Psi$ has at least one fixed point $y_{f}$ and $y_{K_{1} T_{L}} \leq y_{f}<y^{I I}$ (Fig.1(b) and Fig.1(c)). Furthermore, the concavity of $\Psi$ implies the uniqueness of the fixed point $y_{f}$.

(IV) Let the closure of $D$ be

$$
\bar{D}=\left\{(x, y) \mid x>0, y>0, y<\frac{r}{a}\left(1-\frac{x}{K}\right)\right\}
$$

denote

$$
L=y-\frac{r}{a}\left(1-\frac{x}{K}\right) .
$$

If

$$
\left[(P(x, y), Q(x, y)) \cdot\left(\frac{r}{a K}, 1\right)\right]_{L=0}=\left[\frac{b x}{1+w x}-c x-d\right] y<0,
$$

where $\cdot$ is the scalar product of two vectors, then the vector field will finally enter into the boundary $\bar{D}$. It implies that $\bar{D}$ is an invariant set. Concerning property (I), we have $\Psi\left(\left[y_{K_{1} T_{L}},+\infty\right)\right) \subset \Psi\left(\left[0, y_{K_{1} T_{L}}\right]\right)$ which reveals that $\Psi(y)$ is bounded in the definition domain. Note that in the region above the line $L_{1}$ we have $d y / d t<0$ and $d x / d t<0$ and the region below the line $L_{1}$ we have $d y / d t<0$ and $d x / d t>0$, and on the line $x=\left(1-K_{1}\left(C_{0}\right)\right) T_{L}$ the component $y$ satisfies $d y / d t<0$, any trajectory $\psi\left(t ;\left(1-K_{1}\left(C_{0}\right)\right) T_{L}, y_{0}^{+}\right)\left(y_{0}^{+} \in\right.$ $\left.\left(y_{K_{1} T_{L}},+\infty\right)\right)$ will first meet the isocline $L_{1}$, then across $x=\left(1-K_{1}\left(C_{0}\right)\right) T_{L}$ and finally approach the line $x=T_{L}$ after a certain time. These results show that the solution $\psi\left(t ;\left(1-K_{1}\left(C_{0}\right)\right) T_{L}, y_{0}^{+}\right)$satisfies $\lim _{y_{0}^{+} \rightarrow+\infty} \psi\left(t ;\left(1-K_{1}\left(C_{0}\right)\right) T_{L}, y_{0}^{+}\right)=0$. After one single pulse,

$$
\lim _{y_{0}^{+} \rightarrow+\infty} \Psi\left(y_{0}^{+}\right)=\lim _{y_{0}^{+} \rightarrow+\infty}\left(\left(1-K_{2}\left(C_{0}\right)\right) \psi\left(t ;\left(1-K_{1}\left(C_{0}\right)\right) T_{L}, y_{0}^{+}\right)\right) \theta+\tau=\tau .
$$

Therefore, $\Psi$ has a horizontal asymptote $\tau$, Fig.1. This completes the proof.

\section{Order- $k$ periodic solutions}

\subsection{Effector cell eradication periodic solution}

If a patient does not have a strong immunity, then the immune cells in his body will eventually be annihilated by tumour cells. Thus, it is a disaster for the people. To avoid this from happening, we have to show the effects of the key control parameters on the eradications of the effector cells. To this end, we first need to investigate the effector cell eradication periodic solution. Let $y(t)=0$ and $\tau=0$ in system (2) and then we focus on the following subsystem:

$$
\left\{\begin{array}{lrl}
\frac{d x(t)}{d t}=r x(t)\left(1-\frac{x(t)}{K}\right), & x(t)<T_{L}, \\
x\left(t^{+}\right)=\left(1-K_{1}\left(C_{0}\right)\right) x(t), & x(t)=T_{L},
\end{array}\right.
$$

where $K_{1}\left(C_{0}\right)$ represents the treatment term of chemotherapy and is defined by (3). Solving equation (8) with initial condition $x\left(0^{+}\right)=\left(1-K_{1}\left(C_{0}\right)\right) T_{L}$ yields

$$
x^{T}(t)=\frac{\left(1-K_{1}\left(C_{0}\right)\right) T_{L} K \exp (r t)}{K-\left(1-K_{1}\left(C_{0}\right)\right) T_{L}+\left(1-K_{1}\left(C_{0}\right)\right) T_{L} \exp (r t)},
$$


denote the period of this solution by $T$, then solving

$$
\frac{\left(1-K_{1}\left(C_{0}\right)\right) T_{L} K \exp (r T)}{K-\left(1-K_{1}\left(C_{0}\right)\right) T_{L}+\left(1-K_{1}\left(C_{0}\right)\right) T_{L} \exp (r T)}=T_{L}
$$

with respect to $T$, we obtain the expression of the period $T$, i.e.,

$$
T=\frac{1}{r} \ln \frac{K-\left(1-d_{T}\left(1-\exp \left(-k_{1} C_{0}\right)\right) T_{L}\right.}{\left(1-d_{T}\left(1-\exp \left(-k_{1} C_{0}\right)\right)\right)\left(K-T_{L}\right)}, d_{T}=\frac{T_{L}+\left(K-T_{L}\right) \exp (r T)}{\left(K-T_{L}\right)(\exp (r T)-1)\left(1-\exp \left(-k_{1} C_{0}\right)\right)} .
$$

Thereby, the boundary periodic solution of model (2) with period $T$ gives

$$
\left\{\begin{array}{l}
x^{T}(t)=\frac{\left(1-d_{T}\left(1-\exp \left(-k_{1} C_{0}\right)\right)\right) T_{L} K \exp (r(t-(k-1) T))}{K-\left(1-d_{T}\left(1-\exp \left(-k_{1} C_{0}\right)\right)\right) T_{L}+\left(1-d_{T}\left(1-\exp \left(-k_{1} C_{0}\right)\right)\right) T_{L} \exp (r(t-(k-1) T))}, \\
y^{T}(t)=0 .
\end{array}\right.
$$

Theorem 2. The effector cell eradication periodic solution $\left(x^{T}(t), 0\right)$ of system (2) is globally asymptotically stable provided

$$
\begin{aligned}
R_{0} \triangleq & \left(1-K_{2}\left(C_{0}\right)\right) \theta\left(\frac{\left(1-K_{1}\left(C_{0}\right)\right)\left(K-T_{L}\right)}{K-\left(1-K_{1}\left(C_{0}\right)\right) T_{L}}\right)^{\frac{d}{r}}\left(\frac{K-T_{L}}{K-\left(1-K_{1}\left(C_{0}\right)\right) T_{L}}\right)^{\frac{K c}{r}} \\
& \left(\frac{\left(1+w T_{L}\right)\left(K-\left(1-K_{1}\left(C_{0}\right)\right) T_{L}\right)}{\left(K-T_{L}\right)\left(1+w\left(1-K_{1}\left(C_{0}\right)\right) T_{L}\right)}\right)^{\frac{K b}{(1+w) r}}<1,
\end{aligned}
$$

where $K_{1}\left(C_{0}\right)=d_{T}\left(1-\exp \left(-k_{1} C_{0}\right)\right), K_{2}\left(C_{0}\right)=d_{E}\left(1-\exp \left(-k_{2} C_{0}\right)\right)$.

Proof. From Lemma 1, denote

$$
\begin{aligned}
& P(x, y)=r x\left(1-\frac{x}{K}\right)-a x y, \quad Q(x, y)=\frac{b x y}{1+w x}-c x y-d y \\
& \alpha(x, y)=x\left(-K_{1}\left(C_{0}\right)\right), \quad \beta(x, y)=y\left(\left(1-K_{2}\left(C_{0}\right)\right) \theta-1\right)+\tau, \\
& \phi(x, y)=x-T_{L},\left(x^{T}(T), y^{T}(T)\right)=\left(T_{L}, 0\right) \\
& \left(x^{T}\left(T^{+}\right), y^{T}\left(T^{+}\right)\right)=\left(\left(1-K_{1}\left(C_{0}\right)\right) T_{L}, 0\right) .
\end{aligned}
$$

Taking the derivations we obtain

$$
\begin{aligned}
& \frac{\partial P}{\partial x}=r-\frac{2 r x}{K}-a y, \quad \frac{\partial Q}{\partial y}=\frac{b x}{1+w x}-c x-d, \\
& \frac{\partial \alpha}{\partial x}=-K_{1}\left(C_{0}\right), \quad \frac{\partial \beta}{\partial y}=\left(1-K_{2}\left(C_{0}\right)\right) \theta-1, \\
& \frac{\partial \phi}{\partial x}=1, \quad \frac{\partial \alpha}{\partial y}=\frac{\partial \beta}{\partial x}=\frac{\partial \phi}{\partial y}=0,
\end{aligned}
$$

and then

$$
\begin{aligned}
\Delta_{1} & =\frac{P_{+}\left(\frac{\partial \beta}{\partial y} \frac{\partial \phi}{\partial x}-\frac{\partial \beta}{\partial x} \frac{\partial \phi}{\partial y}+\frac{\partial \phi}{\partial x}\right)+Q_{+}\left(\frac{\partial \alpha}{\partial x} \frac{\partial \phi}{\partial y}-\frac{\partial \alpha}{\partial y} \frac{\partial \phi}{\partial x}+\frac{\partial \phi}{\partial y}\right)}{P \frac{\partial \phi}{\partial x}+Q \frac{\partial \phi}{\partial y}} \\
& =\frac{P^{+}\left(x^{T}\left(T^{+}\right), y^{T}\left(T^{+}\right)\right)\left(1+\frac{\partial \beta}{\partial y}\right)}{P\left(x^{T}(T), y^{T}(T)\right)}=\frac{\left(1-K_{2}\left(C_{0}\right)\right) \theta\left(1-K_{1}\left(C_{0}\right)\right)\left(K-\left(1-K_{1}\left(C_{0}\right)\right) T_{L}\right)}{K-T_{L}} .
\end{aligned}
$$

Moreover,

$$
\begin{aligned}
& \int_{0}^{T}\left[\frac{\partial P}{\partial x}\left(x^{T}(t), y^{T}(t)\right)+\frac{\partial Q}{\partial y}\left(x^{T}(t), y^{T}(t)\right)\right] d t \\
& =\int_{0}^{T}\left[r-d-\frac{2 r x^{T}(t)}{K}+\frac{b x^{T}(t)}{1+w x^{T}(t)}-c x^{T}(t)\right] d t \\
& =(r-d) T-\left.\frac{K c+2 r}{r} \ln \left(\frac{\left(1-K_{1}\left(C_{0}\right)\right) T_{L} e^{r t}+K-\left(1-K_{1}\left(C_{0}\right)\right) T_{L}}{K}\right)\right|_{0} ^{T} \\
& +\left.\frac{K b}{r(K w+1)} \ln \left(\frac{(K w+1)\left(1-K_{1}\left(C_{0}\right)\right) T_{L} e^{r t}+K-\left(1-K_{1}\left(C_{0}\right)\right) T_{L}}{K}\right)\right|_{0} ^{T} \\
& =\frac{r-d}{r} \ln \left(\frac{K-\left(1-K_{1}\left(C_{0}\right)\right) T_{L}}{\left(1-K_{1}\left(C_{0}\right)\right)\left(K-T_{L}\right)}\right)+\frac{K c+2 r}{r} \ln \left(\frac{\left(K-T_{L}\right)}{K-\left(1-K_{1}\left(C_{0}\right)\right) T_{L}}\right) \\
& +\frac{K b}{r(K w+1)} \ln \left(\frac{\left(K-\left(1-K_{1}\left(C_{0}\right)\right)_{L}\right)\left(1+w T_{L}\right)}{\left(1+w\left(1-K_{1}\left(C_{0}\right)\right) T_{L}\right)\left(K-T_{L}\right)}\right) .
\end{aligned}
$$

Thus the Floquet multiplier $\mu_{2}$ can be calculated

$$
\begin{aligned}
\left|\mu_{2}\right|= & \Delta_{1} \exp \left(\int_{0}^{T}\left[\frac{\partial P}{\partial x}\left(x^{T}(t), y^{T}(t)\right)+\frac{\partial Q}{\partial y}\left(x^{T}(t), y^{T}(t)\right)\right] d t\right) \\
= & \left(1-K_{2}\left(C_{0}\right)\right) \theta\left(\frac{\left(1-K_{1}\left(C_{0}\right)\right)\left(K-T_{L}\right)}{K-\left(1-K_{1}\left(C_{0}\right)\right) T_{L}}\right)^{\frac{d}{r}}\left(\frac{K-T_{L}}{K-\left(1-K_{1}\left(C_{0}\right)\right) T_{L}}\right)^{\frac{K c}{r}} \\
& \left(\frac{\left(1+w T_{L}\right)\left(K-\left(1-K_{1}\left(C_{0}\right)\right) T_{L}\right)}{\left(K-T_{L}\right)\left(1+w\left(1-K_{1}\left(C_{0}\right)\right) T_{L}\right)}\right)^{\frac{K b}{(1+w K) r}} \triangleq R_{0} .
\end{aligned}
$$


It follows from (11) that $\left|\mu_{2}\right|<1$, which means the effector cell eradication periodic solution $\left(x^{T}(t), 0\right)$ is orbitally asymptotically stable.

In view of the Theorem 1 , for any $i \geq 0$, the impulsive point series $y_{i}^{+} \in\left[0, y_{K_{1} T_{L}}\right]$ is monotonically decreasing on the whole definition domain and finally tends to zero. Thus the effector cell eradication periodic solution $\left(x^{T}(t), 0\right)$ is globally attractive. This completes the proof.

\subsection{Transcritical bifurcation}

\subsubsection{Transcritical bifurcation with respect to killing rate $K_{2}$}

When $\tau=0$, to discuss the occurrence of the transcritical bifurcation, we consider the Poincaré map $\Psi\left(y_{i}^{+}\right)$ defined by (2). For simplicity, assume that $y \geq 0$ be small enough and then let $u=y_{i}^{+}$. So the Poincaré map becomes

$$
u \rightarrow\left(1-K_{2}\left(C_{0}\right)\right) \theta g(u) \equiv F\left(u, K_{2}\right) .
$$

The uniqueness of solutions ensures $g(0)=0$. It follows from continuously differentiable of function $g$ that the function $F(u, q)$ is also continuously differentiable with respect to both $u$ and $K_{2}$, and obviously $\lim _{u \rightarrow 0^{+}} g(u)=g(0)=0$. The following results hold true based on Lemma 2 .

Theorem 3. If $T_{L}<K$, then system (2) exists with a stable order-1 periodic solution when $K_{2} \in\left(K_{2}^{*}-\right.$ $\left.\varepsilon, K_{2}^{*}\right)$ through transcritical bifurcation at $K_{2}=K_{2}^{*}$ with $\varepsilon>0$, where $K_{2}^{*}$ is defined by (11) and satisfies $\left.\mu_{2}\right|_{K_{2}=K_{2}^{*}}=1$.

Proof. To show that the Poincaré map undergoes the transcritical bifurcation, we need to verify that all conditions of Lemma 2 are satisfied. Any solution with initial condition $\left(\left(1-K_{1}\left(C_{0}\right)\right) T_{L}, u\right)\left(0 \leq u \leq u_{0}\right.$, $\left.u_{0} \in D o m\right)$ have intersection point $\left(T_{L}, g(u)\right)$ with $x=E T$. Note that in the area between $L_{2}$ and $y=0$ we have $d x / d t>0$, then system (1) becomes

$$
\frac{d y}{d x}=\frac{Q(x, y)}{P(x, y)}
$$

Let $\left(x, y\left(x ; x_{0}, y_{0}\right)\right)$ be an orbit of system (14), and denote as $x_{0}=\left(1-K_{1}\left(C_{0}\right)\right) T_{L}, y_{0}=u, 0 \leq u \leq u_{0}$, then we get

$$
y\left(x ;\left(1-K_{1}\left(C_{0}\right)\right) T_{L}, u\right)=y(x, u), 0 \leq u \leq u_{0},\left(1-K_{1}\left(C_{0}\right)\right) T_{L} \leq x \leq T_{L},
$$

then,

$$
\frac{\partial y(x, u)}{\partial u}=\exp \left[\int_{\left(1-K_{1}\left(C_{0}\right)\right) T_{L}}^{x} \frac{\partial}{\partial y}\left(\frac{Q(s, y(s, u))}{P(s, y(s, u))}\right) d s\right]
$$

and

$$
\frac{\partial^{2} y(x, u)}{\partial u^{2}}=\frac{\partial y(x, u)}{\partial u} \exp \left[\int_{\left(1-K_{1}\left(C_{0}\right)\right) T_{L}}^{x} \frac{\partial^{2}}{\partial y^{2}}\left(\frac{Q(s, y(s, u))}{P(s, y(s, u))}\right) \frac{\partial y(x, u)}{\partial u} d s\right] .
$$

Clearly, $\partial y(x, u) / \partial u>0$, and

$$
\begin{aligned}
g^{\prime}(0)= & \frac{\partial y\left(T_{L}, 0\right)}{\partial u}=\exp \left[\int_{\left(1-K_{1}\left(C_{0}\right)\right) T_{L}}^{T_{L}} \frac{\partial}{\partial y}\left(\frac{Q(s, y(s, 0))}{P(s, y(s, 0))}\right) d s\right] \\
= & \exp \left[\int_{\left(1-K_{1}\left(C_{0}\right)\right) T_{L}}^{T_{L}} \frac{b s-(c s+d)(1+w s)}{r s(1+w s)\left(1-\frac{s}{K}\right)} d s\right] \\
= & \left(\frac{\left(1-K_{1}\left(C_{0}\right)\right)\left(K-T_{L}\right)}{K-\left(1-K_{1}\left(C_{0}\right)\right) T_{L}}\right)^{\frac{d}{r}}\left(\frac{K-T_{L}}{K-\left(1-K_{1}\left(C_{0}\right)\right) T_{L}}\right)^{\frac{K c}{r}} \\
& \left(\frac{\left(1+w T_{L}\right)\left(K-\left(1-K_{1}\left(C_{0}\right)\right) T_{L}\right)}{\left(K-T_{L}\right)\left(1+w\left(1-K_{1}\left(C_{0}\right)\right) T_{L}\right)}\right)^{\frac{K b}{(1+w K) r}} .
\end{aligned}
$$

Furthermore,

$$
g^{\prime \prime}(0)=g^{\prime}(0) \int_{\left(1-K_{1}\left(C_{0}\right)\right) T_{L}}^{T_{L}} \pi(s) \frac{\partial y(x, 0)}{\partial u} d s,
$$


where

$$
\pi(x)=\frac{2 a x(b x-(c x+d)(1+w x))}{(1+w x)\left(r x\left(1-\frac{x}{K}\right)\right)^{2}}, \quad x \in\left[\left(1-K_{1}\left(C_{0}\right)\right) T_{L}, T_{L}\right] .
$$

In the light of $b-c-d w<2 \sqrt{c w d}$ or $b-c-d w \geq 2 \sqrt{c w d}$ and $x_{2} \geq K$, we have $\pi(x)<0$ when $x \in(0,+\infty)$, which implies $g^{\prime \prime}(0)<0$.

Due to these facts, we now verify conditions of Lemma 2 one by one.

(1) $F\left(0, K_{2}\right)=\left(1-K_{2}\right) \theta g(0)=0$;

(2) Secondly,

$$
\frac{\partial F\left(0, K_{2}\right)}{\partial u}=\left(1-K_{2}\right) \theta g^{\prime}(0)=R_{0},
$$

condition (11) ensures that

$$
\frac{\partial F\left(0, K_{2}^{*}\right)}{\partial u}=1 .
$$

It indicates that $\left(0, K_{2}^{*}\right)$ is a fixed point (with eigenvalue of 1 ) of the map (16);

(3) Thirdly,

$$
\frac{\partial^{2} F\left(0, K_{2}^{*}\right)}{\partial u \partial K_{2}}=\theta g^{\prime}(0)>0,
$$

(4) Finally, follows by $g^{\prime \prime}(0)<0$ we obtain

$$
\frac{\partial^{2} F\left(0, K_{2}^{*}\right)}{\partial u^{2}}=\left(1-K_{2}^{*}\right) \theta g^{\prime \prime}(0)<0 .
$$

Therefore, we have shown that all conditions of Lemma 2 are satisfied. This completes the proof.

Remark. Notice that $K_{2}\left(C_{0}\right)=d_{E}\left(1-\exp \left(-k_{2} C_{0}\right)\right)$, there must be constants $d_{E}^{*}, k_{2}^{*}$ and $C_{0}^{*}$ such that

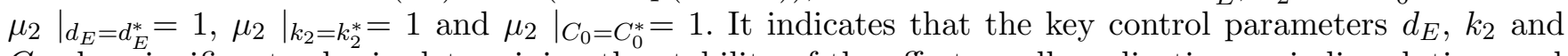
$C_{0}$ play significant roles in determining the stability of the effector cell eradication periodic solution, and these factors also guarantee the occurrence of the transcritical bifurcation near the effector cell eradication periodic solution.

\subsubsection{Transcritical bifurcation with respect to accumulation rate $\theta$}

For the existence of transcritical bifurcation for $\theta^{*}$, defining the Poincaré map as

$$
u \rightarrow\left(1-K_{2}\left(C_{0}\right)\right) \theta g(u) \equiv F(u, \theta) .
$$

Under conditions of $b-c-d w<2 \sqrt{c w d}$ or $b-c-d w \geq 2 \sqrt{c w d}$ and $x_{2} \geq K$, we also obtain that $x \in(0,+\infty)$ reveals $\pi(x)<0$ and $g^{\prime \prime}(0)<0$. Thus, we also examine whether conditions of Lemma 2 holds.

(1) $F(0, \theta)=\left(1-K_{2}\right) \theta g(0)=0$; (2) we have

$$
\frac{\partial F(0, \theta)}{\partial u}=\left(1-K_{2}\right) \theta g^{\prime}(0)=R_{0},
$$

and (11) yields $\frac{\partial F\left(0, \theta^{*}\right)}{\partial u}=1$. where $\theta^{*}$ satisfies $\left.\mu_{2}\right|_{\theta=\theta^{*}}=1$, it implies $\left(0, \theta^{*}\right)$ is a fixed point (with eigenvalue of 1) of (16); (3) $\frac{\partial^{2} F\left(0, \theta^{*}\right)}{\partial u \partial \theta}=\left(1-K_{2}^{*}\right) g^{\prime}(0)>0$, (4) In view of $g^{\prime \prime}(0)<0$,

$$
\frac{\partial^{2} F\left(0, \theta^{*}\right)}{\partial u^{2}}=\left(1-K_{2}\right) \theta g^{\prime \prime}(0)<0 .
$$

Therefore, we have following results.

Theorem 4. If $T_{L}<K$, then system (2) exists with a stable order-1 periodic solution when $\theta \in\left(\theta^{*}, \theta^{*}+\varepsilon\right)$ through transcritical bifurcation at $\theta=\theta^{*}$ with $\varepsilon>0$, where $\theta^{*}$ is defined by (11) and satisfies $\left.\mu_{2}\right|_{\theta=\theta^{*}}=1$.

From above analyses, we find that the transcritical bifurcation near the effector cell eradication periodic solution can occur with respect to many treatment parameters including accumulation rate $\theta$, depletion rate $d_{E}$ of drug toxicity, chemotherapy drug concentration $C_{0}$ and medicine toxicity coefficient $k_{2}$. Meanwhile, the transcritical bifurcation occurs at $\mu_{2}=1$ (or $R_{0}=1$ ) which does not rely on the treatment parameter, with the effector cell eradication periodic solution being stable for $0<\mu_{2}<1$ and unstable for $\mu_{2}>1$. 


\subsection{Periodic solutions when $\tau>0$}

Since the case of $\tau=0$ has been investigated, the coming section deals with order- $k$ periodic solutions when $\tau>0$. As discussed before, any solution with initial point $\left(\left(1-K_{1}\left(C_{0}\right)\right) T_{L}, y_{0}^{+}\right)$will experience finite or infinite many pulses, we let $\Psi^{n}\left(y_{0}^{+}\right)=y_{n}^{+}$be the impulsive point series $y_{n}^{+}(n=1,2, \cdots)$ for simplicity. Theorem 1 reveals that there always exists with an order- 1 periodic solution $(\xi(t), \eta(t))$. By utilizing methods of Theorem 2, we have the following generalized results.

Theorem 5. The order-1 periodic solution $(\xi(t), \eta(t))$ is orbitally asymptotically stable provided

$$
\left|\frac{\left(1-K_{2}\left(C_{0}\right)\right) \theta\left(1-K_{1}\left(C_{0}\right)\right)\left(r\left(1-\frac{\left(1-K_{1}\left(C_{0}\right)\right) T_{L}}{K}\right)-\frac{\left.a\left(\left(1-K_{2}\left(C_{0}\right)\right) \eta_{0}\right) \theta+\tau\right)}{1+w\left(1-K_{1}\left(C_{0}\right)\right) T_{L}}\right)}{r\left(1-\frac{T_{L}}{K}\right)-\frac{a \eta_{0}}{1+w T_{L}}} \exp \left(\int_{0}^{T} G(t) d t\right)\right|<1,
$$

where $G(t)=r-d-\frac{2 r \xi(t)}{K}-(a+c) \eta(t)+\frac{b \xi(t)}{1+w \xi(t)}$.

Proof. Denote the initial point and end point of $(\xi(t), \eta(t))$ by $M\left(T_{L}, \eta_{0}\right)$ and $M^{+}\left(\left(1-K_{1}\left(C_{0}\right)\right) T_{L},((1-\right.$ $\left.\left.\left.K_{2}\left(C_{0}\right)\right) \eta_{0}\right) \theta+\tau\right)$. By calculation we obtain the Floquet multiplier $\mu_{2}$

$$
\begin{aligned}
\mu_{2} & =\Delta_{1} \exp \left(\int_{0}^{T}\left[\frac{\partial P}{\partial x}(\xi(t), \eta(t))+\frac{\partial Q}{\partial y}(\xi(t), \eta(t))\right] d t\right) \\
& =\frac{\left(1-K_{2}\left(C_{0}\right)\right) \theta\left(1-K_{1}\left(C_{0}\right)\right)\left(r\left(1-\frac{\left(1-K_{1}\left(C_{0}\right)\right) T_{L}}{K}\right)-\frac{\left.a\left(\left(1-K_{2}\left(C_{0}\right)\right) \eta_{0}\right) \theta+\tau\right)}{1+w\left(1-K_{1}\left(C_{0}\right)\right) T_{L}}\right)}{r\left(1-\frac{T_{L}}{K}\right)-\frac{a \eta_{0}}{1+w T_{L}}} \exp \left(\int_{0}^{T} G(t) d t\right) .
\end{aligned}
$$

It follows from $\left|\mu_{2}\right|<1$ that the order-1 periodic solution $(\xi(t), \eta(t))$ is always orbitally asymptotically stable. This completes the proof.

Theorem 6. If $\Psi\left(y_{K_{1} T_{L}}\right)<y_{K_{1} T_{L}}$, then system (2) exists with an globally asymptotically stable order-1 periodic solution.

Proof. Due to the property (III) of Theorem 1, if $\Psi\left(y_{K_{1} T_{L}}\right)<y_{K_{1} T_{L}}$, then the Poincaré map $\Psi$ exists with a unique fixed point $y_{f}\left(0<y_{f}<y_{K_{1} T_{L}}\right)$. To show its globally stability, we further need to prove that it is globally attractive.

In the light of properties (II) and (IV) of Theorem 1, giving an initial condition $\left(\left(1-K_{1}\left(C_{0}\right)\right) T_{L}, y_{0}^{+}\right)$, if $y_{0}^{+} \in\left[0, y_{f}\right)$, then $y_{0}^{+}<\Psi\left(y_{0}^{+}\right)<y_{f}$. By induction, $\Psi^{n}\left(y_{0}^{+}\right)$is monotonically increasing and this leads to $\lim _{n \rightarrow+\infty} \Psi^{n}\left(y_{0}^{+}\right)=y_{f}$ (Fig. 1(a)).

In the case of $y_{0}^{+} \in\left(y_{f},+\infty\right)$, if $\Psi^{n}\left(y_{0}^{+}\right)>y_{f}$ holds naturally, then $\Psi\left(y_{0}^{+}\right)<y_{0}^{+}$results in that $\Psi^{n}\left(y_{0}^{+}\right)$ is monotonically decreasing and we have $\lim _{n \rightarrow+\infty} \Psi^{n}\left(y_{0}^{+}\right)=y_{f}$. If $\Psi^{n}\left(y_{0}^{+}\right)>y_{f}$ holds not for all $n$. Thereby, there is a $n_{1}$ such that $\Psi^{n_{1}}\left(y_{0}^{+}\right)<y_{f}$. As discussed above, there is a $n_{2}\left(n_{2}>n_{1}\right)$ such that $\Psi^{n_{2}}\left(y_{0}^{+}\right)$is increasing monotonically, and this results in $\lim _{n_{2} \rightarrow+\infty} \Psi^{n_{2}}\left(y_{0}^{+}\right)=y_{f}$. In a word, the fixed point $y_{f}$ of $\Psi$ is globally attractive. This ends the proof.

Theorem 7. If $\Psi\left(y_{K_{1} T_{L}}\right)>y_{K_{1} T_{L}}$ and $\Psi^{2}\left(y_{K_{1} T_{L}}\right) \geq y_{K_{1} T_{L}}$, then system (2) exists with the order-1 and order-2 periodic solutions.

Proof. If $\Psi\left(y_{K_{1} T_{L}}\right)>y_{K_{1} T_{L}}$, the property (I) of Theorem 1 guarantees that on $\left[0, y_{K_{1} T_{L}}\right]$ the map $\Psi$ is monotonically increasing, which reveals that in this interval there is no fixed point for $\Psi$. Thereby, there must be an constant $i$ such that $y_{i-1}^{+}=\Psi^{n-1}\left(y_{0}^{+}\right)<y_{K_{1} T_{L}}$ and $y_{i}^{+}=\Psi^{n}\left(y_{0}^{+}\right) \geq y_{K_{1} T_{L}}$. On the interval $\left[y_{K_{1} T_{L}},+\infty\right)$, since $\Psi$ is strictly decreasing and $\Psi^{2}$ is strictly increasing,

$$
\Psi\left(\left[y_{K_{1} T_{L}}, \Psi\left(y_{K_{1} T_{L}}\right)\right]\right)=\left[\Psi^{2}\left(y_{K_{1} T_{L}}\right), \Psi\left(y_{K_{1} T_{L}}\right)\right] \subset\left[y_{K_{1} T_{L}}, \Psi\left(y_{K_{1} T_{L}}\right)\right] .
$$

It indicates that $\left[y_{K_{1} T_{L}}, \Psi\left(y_{K_{1} T_{L}}\right)\right]$ is an invariant set for $\Psi^{n}\left(y_{0}^{+}\right)$. Hence, the fixed points of $\Psi$ relies on the initial value of $y_{0}^{+}$. For any $y_{0}^{+} \in\left[y_{K_{1} T_{L}}, \Psi\left(y_{K_{1} T_{L}}\right)\right]$, we denote $y_{1}^{+}=\Psi\left(y_{0}^{+}\right) \neq y_{0}^{+}, y_{2}^{+}=\Psi^{2}\left(y_{0}^{+}\right) \neq y_{0}^{+}$. If $y_{1}^{+}=\Psi\left(y_{0}^{+}\right)=y_{0}^{+}$and $y_{2}^{+}=\Psi^{2}\left(y_{0}^{+}\right)=y_{0}^{+}$, then $y_{0}^{+}$is just the fixed point of $\Psi$. Otherwise, for the relations among $y_{P}, \Psi\left(y_{P}\right), y_{0}^{+}, y_{1}^{+}$and $y_{2}^{+}$, there are four cases: 
$\left(C_{1}\right)$ If $y_{K_{1} T_{L}} \leq y_{2}^{+}<y_{0}^{+}<y_{1}^{+} \leq \Psi\left(y_{K_{1} T_{L}}\right)$, then $y_{1}^{+}=\Psi\left(y_{0}^{+}\right)<\Psi\left(y_{2}^{+}\right)=y_{3}^{+}$and $y_{4}^{+}=\Psi\left(y_{3}^{+}\right)<\Psi\left(y_{1}^{+}\right)=$ $y_{2}^{+}$. Thus $y_{4}^{+}<y_{2}^{+}<y_{0}^{+}<y_{1}^{+}<y_{3}^{+}$. By induction we have

$$
\begin{aligned}
& y_{K_{1} T_{L}} \leq \cdots<y_{2 n+2}^{+}<y_{2 n}^{+}<\cdots<y_{2}^{+}<y_{0}^{+} \\
& <y_{1}^{+}<\cdots<y_{2 n-1}^{+}<y_{2 n+1}^{+}<\cdots \leq \Psi\left(y_{K_{1} T_{L}}\right) .
\end{aligned}
$$

$\left(C_{2}\right)$ If $y_{K_{1} T_{L}} \leq y_{0}^{+}<y_{2}^{+}<y_{1}^{+} \leq \Psi\left(y_{K_{1} T_{L}}\right)$, then $\Psi\left(y_{1}^{+}\right)=y_{2}^{+}<y_{3}^{+}=\Psi\left(y_{2}^{+}\right)<\Psi\left(y_{0}^{+}\right)=y_{1}^{+}$and $y_{2}^{+}=\Psi\left(y_{1}^{+}\right)<\Psi\left(y_{3}^{+}\right)=y_{4}^{+}<y_{3}^{+}=\Psi\left(y_{2}^{+}\right)=y_{1}^{+}$, i.e., $y_{0}^{+}<y_{2}^{+}<y_{4}^{+}<y_{3}^{+}<y_{1}^{+}$. By induction

$$
\begin{aligned}
& y_{K_{1} T_{L}} \leq y_{0}^{+}<y_{2}^{+}<\cdots<y_{2 n}^{+}<y_{2 n+2}^{+} \\
& <\cdots<y_{2 n+1}^{+}<y_{2 n-1}^{+}<\cdots<y_{1}^{+} \leq \Psi\left(y_{K_{1} T_{L}}\right) .
\end{aligned}
$$

$\left(C_{3}\right)$ If $y_{K_{1} T_{L}} \leq y_{1}^{+}<y_{2}^{+}<y_{0}^{+} \leq \Psi\left(y_{K_{1} T_{L}}\right)$, as shown in case $\left(C_{2}\right)$,

$$
\begin{aligned}
& y_{K_{1} T_{L}} \leq y_{1}^{+}<\cdots<y_{2 n-1}^{+}<y_{2 n+1}^{+}<\cdots \\
& <y_{2 n+2}^{+}<y_{2 n}^{+}<\cdots<y_{2}^{+}<y_{0}^{+} \leq \Psi\left(y_{K_{1} T_{L}}\right) .
\end{aligned}
$$

$\left(C_{4}\right)$ If $y_{K_{1} T_{L}} \leq y_{1}^{+}<y_{0}^{+}<y_{2}^{+} \leq \Psi\left(y_{K_{1} T_{L}}\right)$, as shown in case $\left(C_{1}\right)$

$$
\begin{aligned}
& y_{K_{1} T_{L}} \leq \cdots<y_{2 n+1}^{+}<y_{2 n-1}^{+}<\cdots<y_{1}^{+}<y_{0}^{+} \\
& <y_{2}^{+}<\cdots<y_{2 n}^{+}<y_{2 n+2}^{+}<\cdots \leq \Psi\left(y_{K_{1} T_{L}}\right) .
\end{aligned}
$$

For cases $\left(C_{2}\right)$ and $\left(C_{3}\right)$, because $\Psi^{2 n}\left(y_{0}^{+}\right)=y_{2 n}^{+}$is strictly increasing and $\Psi^{2 n+1}\left(y_{0}^{+}\right)=y_{2 n+1}^{+}$is strictly decreasing, there must be a positive constant $y_{f}$ such that

$$
\lim _{n \rightarrow+\infty} y_{2 n+1}^{+}=\lim _{n \rightarrow+\infty} y_{2 n}^{+}=y_{f}, \quad y_{f} \in\left[y_{K_{1} T_{L}}, \Psi\left(y_{K_{1} T_{L}}\right)\right] .
$$

For case $\left(C_{1}\right)$ and $\left(C_{4}\right)$, there are two positive constants $y_{f}^{*}$ and $y_{f_{*}}$ such that

$$
\lim _{n \rightarrow+\infty} y_{2 n+1}^{+}=y_{f}^{*} \quad \text { and } \lim _{n \rightarrow+\infty} y_{2 n}^{+}=y_{f_{*}},
$$

where $y_{f}^{*}, y_{f_{*}} \in\left[y_{K_{1} T_{L}}, \Psi\left(y_{K_{1} T_{L}}\right)\right]$ and $y_{f}^{*} \neq y_{f_{*}}$. All these results implies that system (2) exists with the order-1 and order-2 periodic solutions (Fig.1 or Fig.2). This completes the proof.

(a)

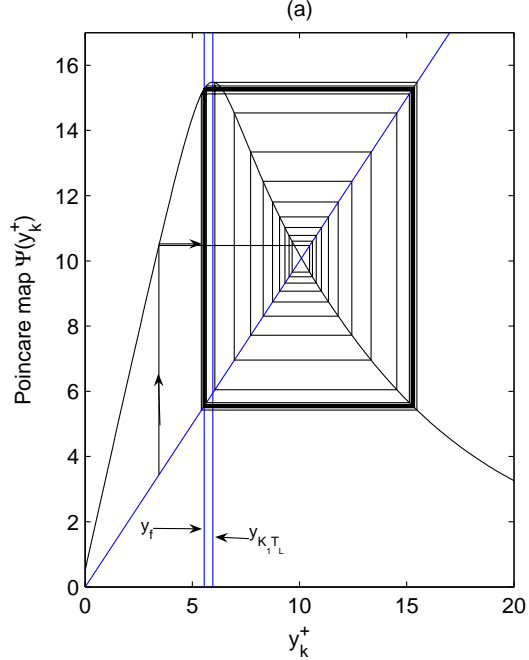

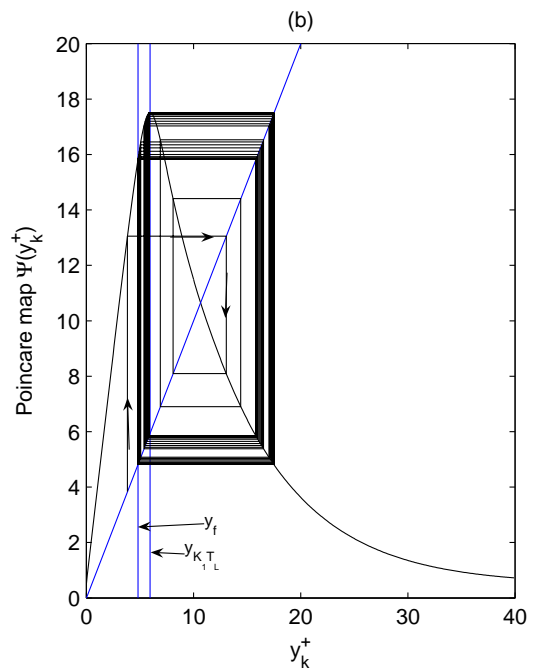

Fig. 2. Fixed points of Poincaré map $\Psi$. (a) Period-2 fixed points for $\theta=3.7$; (b) Period-4 fixed points for $\theta=4.2$. All other parameters were fixed as $r=5, K=100, a=0.8, w=1, b=0.3, c=0.23, d=0.3, d_{T}=0.2, d_{E}=0.4, k_{1}=0.5, k_{2}=1$, $C_{0}=0.65, \tau=0.5$ and $E T=0.5$.

Theorem 8. Denote $y_{\min }^{+}=\min \left\{y^{+}: \Psi\left(y^{+}\right)=y_{K_{1} T_{L}}\right\}$. If $\Psi\left(y_{K_{1} T_{L}}\right)>y_{K_{1} T_{L}}$ and $\Psi^{2}\left(y_{K_{1} T_{L}}\right)<y_{\min }^{+}$, then system (2) exists with an order-3 periodic solution. 
Proof. If $\Psi\left(y_{K_{1} T_{L}}\right)>y_{K_{1} T_{L}}$, in the light of Theorem 1 and Theorem 7 , on $\left(y_{K_{1} T_{L}}, \Psi\left(y_{K_{1} T_{L}}\right)\right)$ there exists a unique fixed point $y_{f}$ for $\Psi$. Theorem 1 also ensures that $\Psi^{3}(y)$ is also continuous on $[0,+\infty)$, then for $\Psi^{3}$ we have: (I) $\Psi^{3}$ is increasing on $\left[0, y_{\min }\right)$ and $\Psi^{3}(0)=\Psi^{2}(\tau)>0$; (II) $\Psi^{3}\left(y_{\min }^{+}\right)=\Psi^{2}\left(y_{K_{1} T_{L}}\right)<y_{\min }^{+}$. Due to continuity of $\Psi^{3}$, it follows from the intermediate value theorem that there is a constant $\bar{y} \in\left(0, y_{\min }^{+}\right)$ such that $\Psi^{3}(\bar{y})=\bar{y}$ and $y_{\min }^{+}<y_{K_{1} T_{L}}$. Moreover, $y_{f} \in\left(y_{K_{1} T_{L}}, \Psi\left(y_{K_{1} T_{L}}\right)\right)$ and thus $\bar{y} \neq y_{f}$. All these results confirm that there is a fixed point $\bar{y} \in\left(0, y_{\min }^{+}\right)$for $\Psi^{3}$. This completes the proof.

\section{Numerical results and biological implications}

In this section, we first carry out sensitivity analyses to show how key control parameter values affect the efficacy of chemotherapeutic drug and outcomes of tumours. Then we choose the control parameters representing the treatment intensity as the bifurcation parameters and provide one-dimensional bifurcations to address the complexity of system (2). Meanwhile, biological conclusions are also presented.

\subsection{Sensitivity analyses}

(a)

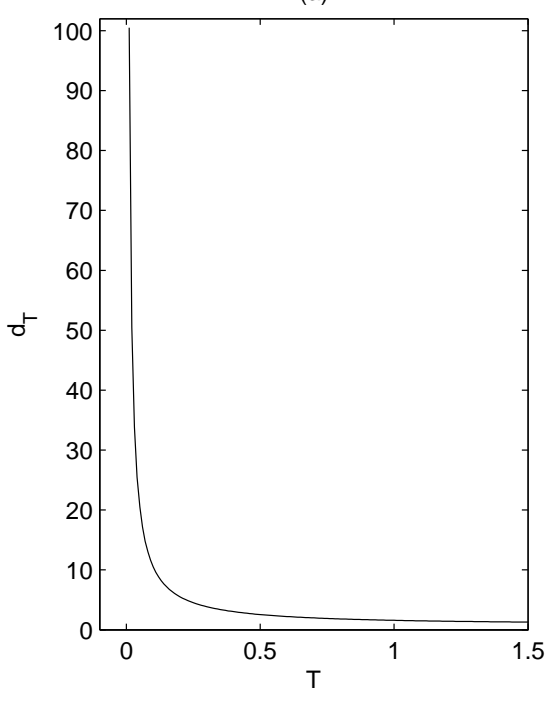

(b)

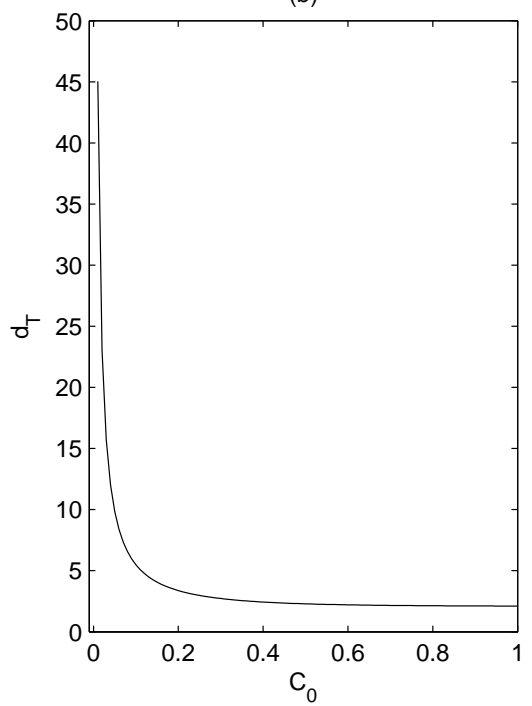

Fig. 3. The effects of key parameters on $d_{T}$ of chemical control. (a) $r=5, K=100, k_{1}=1, C_{0}=0.65$ and $T_{L}=5$; (b) $r=5, K=100, T=10, k_{1}=1$ and $T_{L}=5$.

Since we derive the expression of the effector cell eradication periodic solution in section (4.1), this enable us to explore the effects of period and dosage of chemotherapeutic drugs on the efficacy of chemotherapy. Based on (9) and fixed the parameters, the results indicate that the tumour cell depletion rate $d_{T}$ is decreasing when period $T$ (or chemotherapy drug concentration $C_{0}$ ) is increasing, and the trend is more profound for a smaller $T$ or $C_{0}$ (Fig.3). It is concluded that the therapeutic regimen with a smaller chemotherapy drug dose and more frequent intakes is more effective to maintain higher tumour cell depletion rate (i.e., killing rate of tumours). Thus, smaller period $T$ and lower concentration $C_{0}$ make great contributions to the efficacy of chemotherapy.

Threshold condition (11) is a very important criterion: if $R_{0}<1$, then the effector cell eradication period solution is globally stable; if $R_{0}=1$, then the effector cell eradication period solution becomes unstable; if $R_{0}>1$, then the tumours and effector cells coexist along an interior periodic solution which is generated from transcritical bifurcation. Biologically, it is of great importance to analyze the effects of each control parameters against $R_{0}$. From Fig.4(a), $R_{0}$ decreases as $d_{E}$ increases. For fixed $d_{E}\left(d_{E}=1.35\right)$, $R_{0}$ first increases and then decreases as threshold $T_{L}$ increases. Particularly, $R_{0}<1$ for a small threshold $T_{L}, R_{0} \geq 1$ once $T_{L}$ exceeds a critical value and again $R_{0}<1$ as $T_{L}$ further increases. $R_{0}$ also decreases as 
(a)

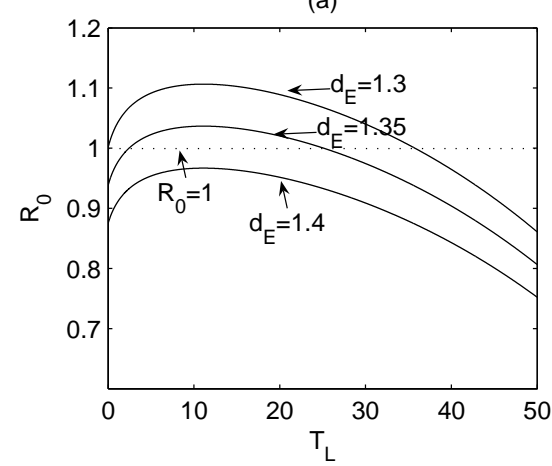

(c)

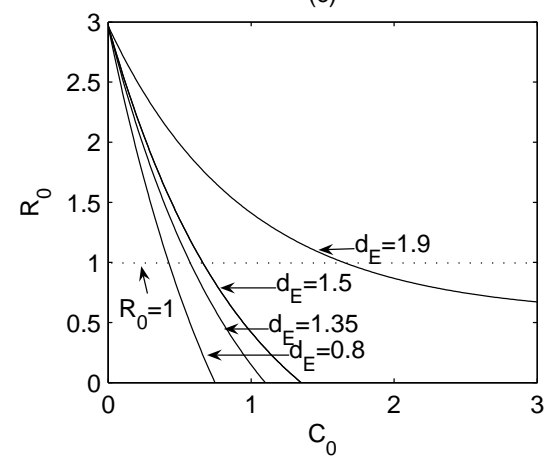

(b)

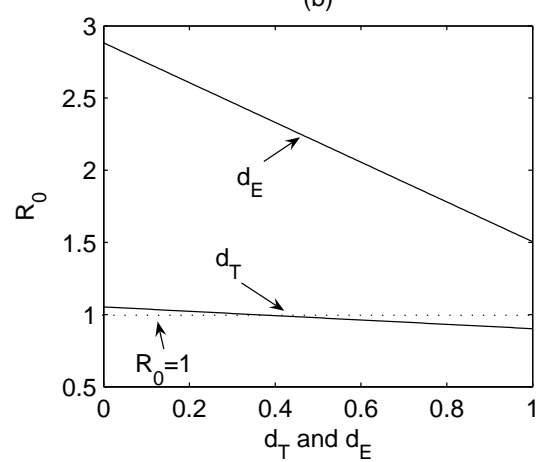

(d)

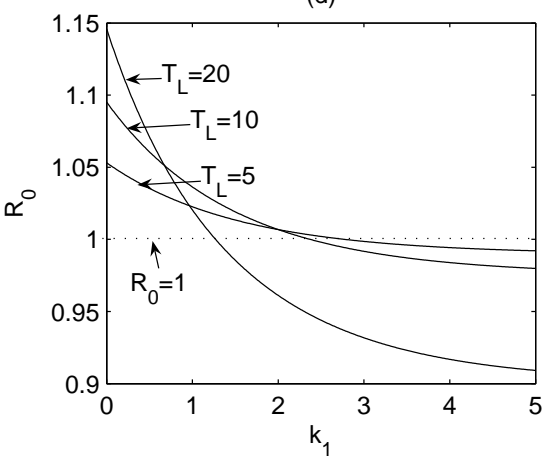

Fig. 4. The effects of control parameters on the threshold condition $R_{0}$. All baseline parameter values were fixed as $r=5$, $K=100, a=0.8, w=1, b=0.3, c=0.23, d=0.3, k_{1}=k_{2}=1, C_{0}=0.65, d_{T}=0.2, d_{E}=1.35, \theta=3.5, E T=5$ and $\tau=0$.

$d_{T}$ increases (Fig.4(b)). $R_{0}$ decreases from $R_{0} \geq 1$ to $R_{0}<1$ as $C_{0}$ and $k_{1}$ increase (Fig.4(c) and Fig.4(d)). The differences are that the trends are more significant for a smaller $d_{E}$ in Fig.4(c) and for a larger $T_{L}$ in Fig.4(d). From a biological view of point, for a single chemotherapy, to control tumours the feasible ways include: increasing $d_{E}$, or increasing $d_{T}$, or decreasing $d_{E}$ and increasing $T_{L}$, or decreasing $d_{E}$ and increasing $C_{0}$, or increasing $k_{1}$ and increasing $T_{L}$. Therefore, the controllable treatment parameters of $d_{E}$, $d_{T}, T_{L}, C_{0}$ and $k_{1}$ play decisive roles in maintaining the tumours below a given threshold.

Though sensitive analysis reveals that mall changes of these factors will result in the sharp changes in the evolution of tumours, the deeper influence of these elements on the dynamics still needs to be explored. To do this, we will carry out a one-dimensional bifurcation analysis with respect to key parameters, because it not only shows the innate dynamical properties of the proposed system, but also indicates how dynamics rely on the parameter.

\subsection{Bifurcation analysis}

In subsections (4.1) and (4.2), the effector cell eradication periodic solution is stable provided $0<\mu_{2}<1$ and unstable provided $\mu_{2}>1$, and the transcritical bifurcation near this periodic solution occurs at $\mu_{2}=1$ (or $R_{0}=1$ ) with respect to the treatment parameters $d_{E}, \theta, k_{2}$ and $C_{0}$. Fixed parameter values as shown in Fig.5(a), if $d_{E}>d_{E}^{*} \approx 1.53$, then we observe a stable effector cell eradication periodic solution, the stability changes from stable to unstable and also the transcritical bifurcation occurs at $d_{E}=d_{E}^{*}$ which subsequently generates an order-1 periodic solution. As $d_{E}$ decreases, the order-1 periodic solution becomes unstable and then there is an stable order-2 periodic solution. When the intensity of immunotherapy is enhanced, i.e., the dosage of external input of IL-2 increases from $\theta=3.5$ to $\theta=7$ (Fig.5(b)), then $d_{E}^{*} \approx 1.832$. It is found that the transcritical bifurcation occurs at $d_{E}^{*}$, then the period-doubling bifurcations leading system (2) to chaos as $d_{E}$ decreases. Meanwhile, complex dynamics includes chaotic bands, periodic windows, crisis, period-halving bifurcations were also observed. Biologically, the depletion rate $d_{E}$ and accumulation rate 

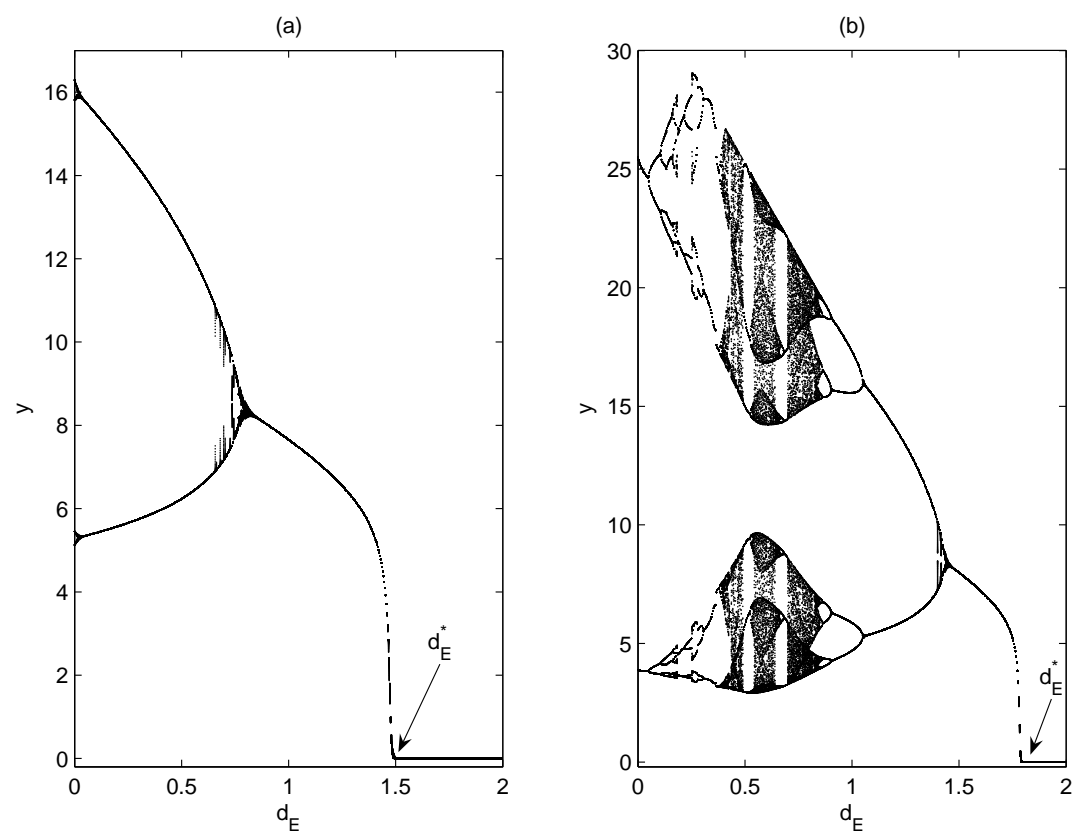

Fig. 5. Bifurcation diagrams with respect to $d_{E}$ when $(K, 0)$ is globally stable with parameters fixed as: $r=5, K=100$, $a=0.8, w=1, b=0.3, c=0.23, d=0.3, k_{1}=k_{2}=1, C_{0}=0.65, d_{T}=0.2, E T=5$ and $\tau=0$. (a) $\theta=3.5$; (b) $\theta=7$.

$\theta$ play significant roles in tumour treatment, small $d_{E}$ and $\theta$ will stabilize the tumour and effector cells along periodic solutions and so will make the treatment more stable.

It follows from subsection (4.2.2) that system (2) will also undergo the transcritical bifurcation with respect to parameter $\theta$. Fix parameter values as shown in Fig.6(a), we obtain $\theta^{*} \approx 1.115$, when $0<\theta<\theta^{*}$, there is an unstable effector cell free periodic solution. As $\theta$ increases, the transcritical bifurcation occurs at $\theta^{*}$ and then period-doubling bifurcations lead to chaos. When $\tau>0$, in subsection (4.3) we have shown that system (2) exists with periodic solutions of any period, the numerical investigations confirm our results. For example, let $\tau=0.5$ and fix all parameters as shown in Fig.6(a), then system (2) exhibits complexity includes period-doubling bifurcations, period-halving bifurcations, etc. Moreover, in the case of $\tau>0$, if we choose $d_{E}$ and $\tau$ as bifurcation parameters, then chaos can be observed and period-having bifurcations lead system (2) from chaos to an stable order-1 periodic solution (7).

In a word, the value of $\mu_{2}$ (or $R_{0}$ ) can be viewed as a key criterion for the stability of the effector cell eradication periodic solution, which was greatly affected by treatment parameters $d_{E}, d_{T}, T_{L}, C_{0}$ and $k_{1}$. The introduce of immunotherapy has contributed greatly to the coexist of tumours and effector cells, as indicated by periodic solutions or chaos. These results indicate that immunotherapy plays a decisive role in evolution of tumours.

\section{Conclusions}

Using mathematical models to study the inner relationships between tumour cells and immune cells and the effects of various treatments on the evolution of tumours have always been one of the hot topics [Walther \& Lin, 2013; Yang et al., 2015, 2019a,b; Tang et al., 2015, 2017]. For tumour-immune systems with control, many factors affecting the outcomes of treatment have been extensively studied, such as surgery, chemotherapy, radiotherapy, immunotherapy or their combinations [Kirschner \& Panetta, 1998; de Pillis et al., 2006; Walther \& Lin, 2013; Yang et al., 2015; Tang et al., 2015, 2017]. While the depletion rates of tumours and immune cells caused by treatment are often assumed to be constants. To study the effects of key factors of treatment on the evolution of tumours more profoundly, we proposed a novel tumour-immune model with pulsed chemotherapy and immunotherapy concerning a tumour threshold value $T_{L}$. 

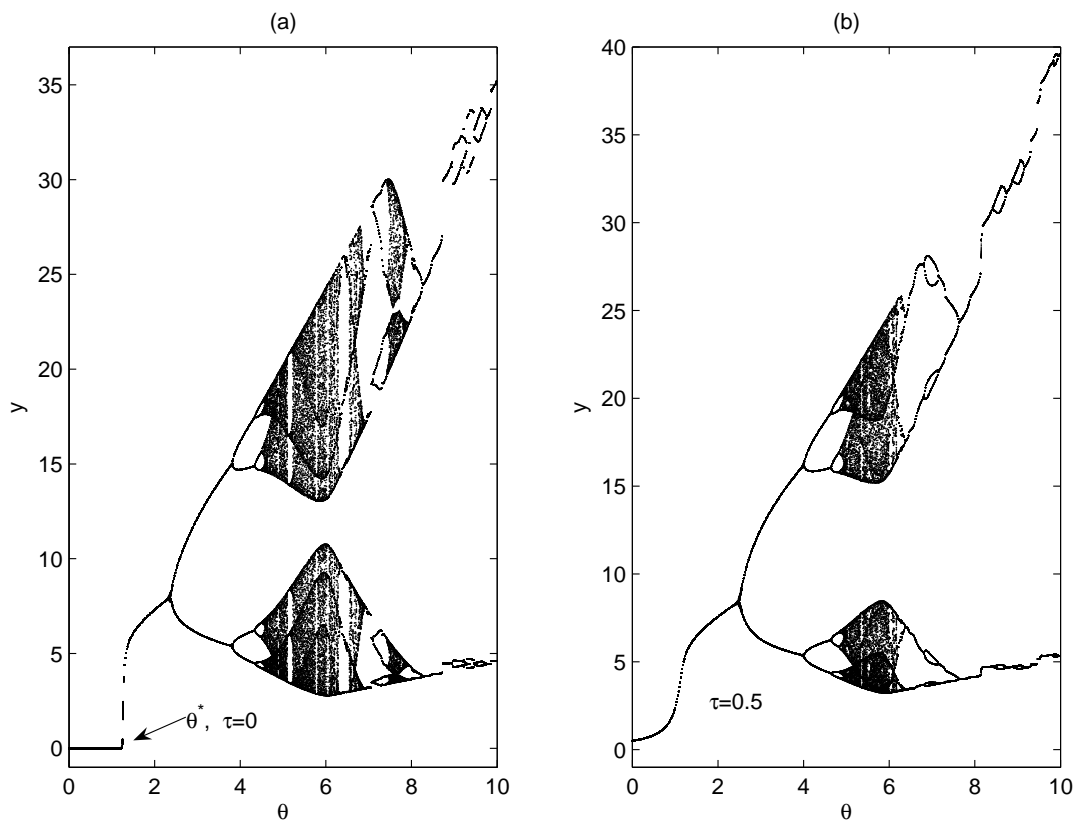

Fig. 6. (a) Bifurcation diagrams with respect to $\theta$ and when $\tau=0$; (b) Bifurcation diagrams with respect to $\theta$ and when $\tau=0.5$. All other parameters are fixed as: $r=5, K=100, a=0.8, w=1, b=0.3, c=0.23, d=0.3, k_{1}=0.5, k_{2}=1$, $C_{0}=0.65, d_{T}=0.2, d_{E}=0.4$ and $E T=5$.
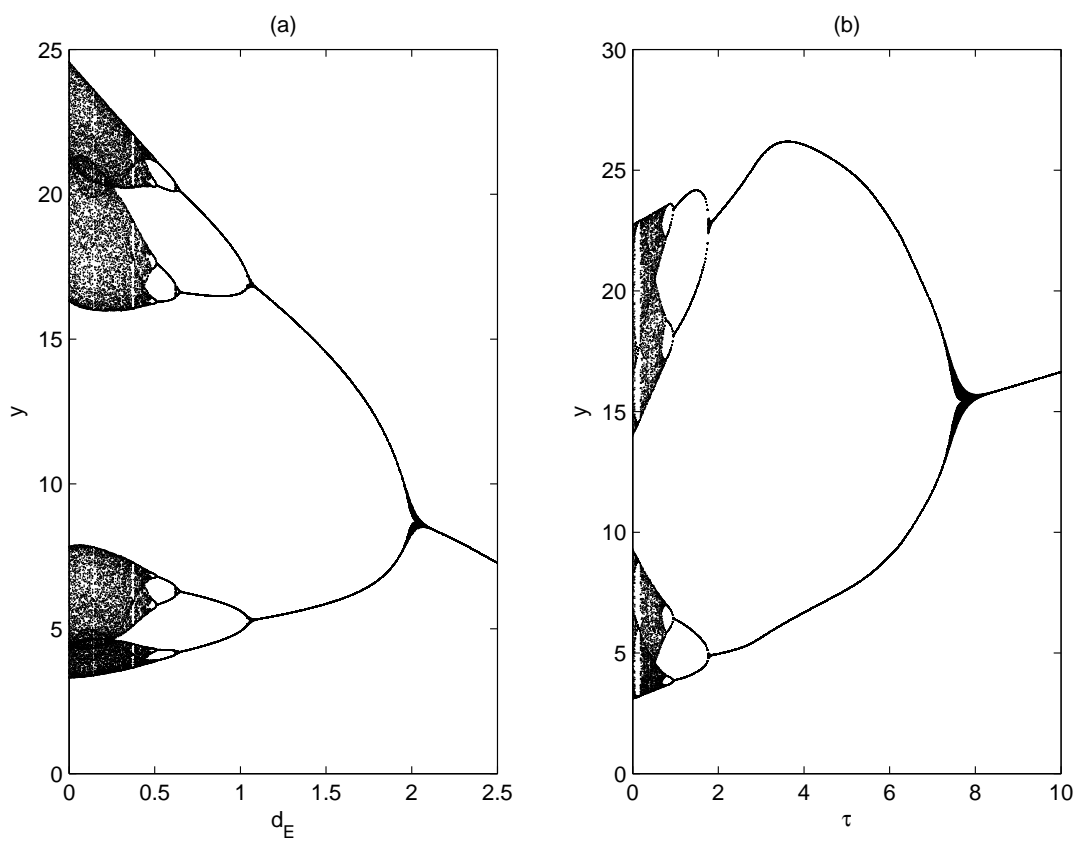

Fig. 7. (a)Bifurcation diagrams with respect to $d_{E}$ when $\tau=0.5$; (b) Bifurcation diagrams with respect to $\tau$ when $d_{E}=0.2$. All other parameters are fixed as: $r=5, K=100, a=0.8, w=1, b=0.3, c=0.23, d=0.3, k_{1}=0.5, k_{2}=0.5, C_{0}=0.65$, $d_{T}=0.3, \theta=5$ and $E T=5$.

First of all, the Poincaré map is defined in the domain and some important properties are discussed. Then we derive the expression of effector cell eradication periodic solution, and obtain the expressions of the period $T$ and the depletion rate $d_{T}$. Threshold condition for the globally asymptotically stability of 
this periodic solution is also provided. It indicates that the key control parameters play significant roles in determining the stability of the effector cell eradication periodic solution, and transcritical bifurcations with respect to the accumulation rate, the depletion rate, medicine toxicity coefficient and the concentration of chemotherapy are also addressed. When immunotherapy (i.e., $\tau>0$ and $\theta>0$ ) is initiated, we study the order- $k(k \geq 1)$ periodic solutions and give conditions for its stability. The results show that a single chemotherapy may stabilize the tumours along the effector cell eradication periodic solution under certain conditions, while tumours and immune cells may coexist along order- $k$ periodic solutions when immunotherapy is introduced.

Furthermore, sensitivity analyses are carried out to show how key treatment parameter values affect the efficacy of chemotherapeutic drug and outcomes of tumours. We find that the tumour cell depletion rate $d_{T}$ is decreasing when impulsive period $T$ or chemotherapy drug concentration $C_{0}$ is increasing, and the trend is more profound for a smaller $T$. It indicates that the therapeutic regimen with a smaller chemotherapy drug dose and more frequent intakes make great contributions to the efficacy of chemotherapy. For a single chemotherapy, the feasible treatment for cancer is increasing the depletion rate $d_{E}$ of effector cell, or increasing $d_{T}$, or decreasing $d_{E}$ and increasing tumour cell threshold $T_{L}$, or decreasing $d_{E}$ and increasing $C_{0}$, or increasing medicine toxicity coefficient $k_{1}$ and increasing $T_{L}$. Therefore, the treatment parameters $d_{E}$, $d_{T}, T_{L}, C_{0}$ and $k_{1}$ play decisive roles in maintaining the tumours below a given threshold. At the same time, treatments with different types of chemotherapeutic drugs, an appropriate dosage, a reasonable impulsive period and a critical tumour cell threshold have significant impacts on the outcomes of cancer treatment. Moreover, the transcritical bifurcation occurs at $R_{0}=1$ with respect to the treatment parameters $d_{E}, \theta, k_{2}$ and $C_{0}$, bifurcation analyses show that many complex dynamics includes chaotic bands, periodic windows, crisis, period-halving bifurcations can be observed when these parameters changes. The results reveal that the introduce of immunotherapy has contributed greatly to the coexist of tumours and effector cells, as indicated by periodic solutions or chaos.

Now, we show some of the highlights of this study compared to previous studies with pulsed treatments [Tang et al., 2016]: (1) the depletion rates or reductions of chemotherapy are usually being constants, but we consider the dose responses as nonlinear pulses in the model (2); (2) transcritical bifurcations with respect to the accumulation rate, the depletion rate, medicine toxicity coefficient and the concentration of chemotherapy are investigated; (3) we address the importance of the key treatment parameter values on the efficacy of chemotherapeutic drug and outcomes of tumours; (4) system (2) has very complex dynamics with respect to not only parameters of chemotherapy, but also parameters of immunotherapy.

For the tumour-immune model, we consider the effects of the dose responses on tumours and immune cells as nonlinear pulses. A very important assumption is that the tumour cells and effector cells are followed by deterministic laws. However, almost all of natural phenomena undergo stochastic processes rather than only by strictly deterministic laws [Renshaw , 1995]. For example, the conditions of the environment (such as temperature, $\mathrm{pH}$ value, nutrition and so on) may change the enzymatic activity of the proteins, and finally have impacts on the evolution of tumours [Caravagna et al., 2010]. Future work is required to consider both pulsed treatment and environmental noise in the tumour-immune systems.

\section{Acknowledgments}

This work was supported by the Chongqing Science and Technology Commission (cstc2017jcyjAX0131, cstc2016jcyjA0412) and the National Natural Science Foundation of China (NSFC:11801047, 11761031).

\section{References}

Bainov, D. \& Simeonov, P. [1993] Impulsive Differential Equations: Periodic Solutions and Applications, vol. 66, Longman.

Caravagna, G., dOnofrio, A., Milazzo, P. \& Barbuti, R. [2010] "Tumour suppression by immune system through stochastic oscillations", J. Theoret. Biol. 265, 336-345.

Ciesielski, K. [2004] "On time reparametrizations and isomorphisms of impulsive dynamical systems", Ann. Polon. Math. 84, 1-25. 
de Pillis, L.G., Gu, W. \& Radunskaya, A. [2006] "Mixed immunotherapy and chemotherapy of tumours: modeling, applications and biological interpretations", J. Theoret. Biol. 238, 841-862.

Dearnaley, D.P., Khoo, V.S., Norman, A.R., Meyer, L., Nahum, A., Tait, D., Yarnold, J. \& Horwich, A. [1999] "Comparison of radiation side-effects of conformal and conventional radiotherapy in prostate cancer: a randomised trial", Lancet, 353, 267-272.

Duffey, B. G., Choyke, P. L., Clenn, G., Grubb, R. L., Venzon, D., Linehan, W. M. \& Walther, M. M. [2004] "The relationship between renal tumor size and metastases in patients with von HippelCLindau disease", J. Urol. 172, 63-65.

Finkelstein, S.E. \& Fishman, M. [2012] "Clinical opportunities in combining immunotherapy with radiation therapy", Front. Oncol. 2, 169.

Gubin,M. M., Zhang, X.L., Schuster,H. et al. [2014] "Checkpoint blockade cancer immunotherapy targets tumour-specific mutant antigens", Nature, 545, 577-593.

Hegmans, J. P., Hemmes, A., Aerts, J. G., Hoogsteden, H. C. \& Lambrecht, B. N. [2005] "Immunotherapy of murine malignant mesothelioma using tumor lysatepulsed dendritic cells", Am. J. Respir. Crit. Care Med. 171, 1168-1177.

Kaul, S. [1990] "On impulsive semi-dynamical systems", J. Math. Anal. Appl. 150, 120-128.

Kirschner, D. \& Panetta, J.C. [1998] "Modeling immunotherapy of the tumour-immune interaction", $J$. Math. Biol. 37, 235-252.

Kuznetsov, V.A., Makalkin, I.A., Taylor, M.A. \& Perelson, A.S. [1994] "Nonlinear dynamics of immunogenic tumours: parameter estimation and global bifurcation analysis", Bull. Math. Biol. 56, 295-321.

Pazdur, R., Hoskins, W., Wagman, L. \& Coia, L. [2005] Cancer Management: A Mulitdisciplinary Approach, eighth edition, Oncology Publishing Group of CMP Healthcare Media, Available at http://www.cancernetwork.com/ handbook/contents.htm.

Panetta, J.C. [1996] "A mathematical model of periodically pulsed chemotherapy: tumor recurrence and metastasis in a competitive environment", Bull. Math. Biol. 58, 425-447.

Rasband, S. [1990] Chaotic Dynamics of Nonlinear Systems, J. Wiley and Sons, New York.

Renshaw, E. [1995] Modelling Biological Population in Space and Time, Cambridge University Press, Cambridge.

Ribas, A., Butterfield, L.H., Glaspy, J.A. \& Economou, J.S. [2003] "Current developments in cancer vaccines and cellular immunotherapy", J. Clin. Oncol. 21, 2415-2432.

Shu, H.Y., Wang, L. \& Watmough, J. [2014] "Sustained and transient oscillations and chaos induced by delayed antiviral immune response in an immunosuppressive infection model", J. Math. Biol. 68, $477-503$.

Simeonov, P. \& Bainov, D. [1988] "Orbital stability of periodic solutions of autonomous systems with impulsive effect", Internat. J. Systems. Sci. 19, 2561-2585.

Tang,B., Xiao,Y.N., Cheke, R.A.\& Wang, N. [2015] "Piecewise virus-immune dynamic model with HIV-1 RNA-guided therapy", J. Theor. Biol. 377, 36-46.

Tang, B., Xiao, Y.N., Sivaloganathan, S. \& Wu, J.H. [2017] "A piecewise model of virus-immune system with effector cell-guided therapy", Appl. Math. Model. 47, 227-248.

Tang, B., Xiao, Y.N., Tang, S.Y. \& Cheke, R.A. [2016] "A feedback control model of comprehensive therapy for treating immunogenic tumours", Int. J. Bifurcat. Chaos. 26, 1650039.

Walther, M. M., Choyke, P. L., Glenn, G., Lyne, J. C., Rayford, W., Venzon, D. \& Linehan, W. M. [1999] "Renal cancer in families with hereditary renal cancer: Prospective analysis of a tumor size threshold for renal parenchymal sparing surgery", J. Urol. 161, $1475 \mathrm{C} 1479$.

Wei, H. \& Lin, J. [2013] "Periodically pulsed immunotherapy in a mathematical model of tumour-immune interaction", Int. J. Bifurcation Chaos. 23, 1350068.

Yamaguchi, S., Ogiue-Ikeda, M., Sekino \& M. Ueno, S. [2006] "Effects of pulsed magnetic stimulation on tumor development and immune functions in mice", Bioelectromagnetics, 27, 64-72.

Yang, J., Tang, S.Y. \& Cheke, R.A. [2015] "Modelling pulsed immunotherapy of tumour-immune interaction", Math. Comput. Simulat. 109, 92-112.

Yang, J., Tan, Y.S. \& Cheke, R.A. [2019a] "Thresholds for extinction and proliferation in a stochastic tumour-immune model with pulsed comprehensive therapy", Nonlinear. Sci. Numer. Simulat. 73, 
363-378.

Yang, J., Tan, Y.S. \& Cheke, R.A. [2019b] "Modelling effects of a chemotherapeutic dose response on a stochastic tumour-immune model", Chaos. Soliton. Fract. 123, 1-13. 